ARTICLE

https://doi.org/10.1038/s41467-018-07855-x

\title{
The ASCIZ-DYNLL1 axis promotes 53BP1- dependent non-homologous end joining and PARP inhibitor sensitivity
}

Jordan R. Becker', Raquel Cuella-Martin', Marco Barazas², Rui Liu³, Catarina Oliveira (D) 1, Antony W. Oliver (D) 4, Kirstin Bilham ${ }^{1}$, Abbey B. Holt ${ }^{5}$, Andrew N. Blackford ${ }^{6,7}$, Jörg Heierhorst (10 ${ }^{3,8}$, Jos Jonkers², Sven Rottenberg (1) ${ }^{2,9} \&$ J. Ross Chapman (1) ${ }^{1}$

53BP1 controls a specialized non-homologous end joining (NHEJ) pathway that is essential for adaptive immunity, yet oncogenic in BRCA1 mutant cancers. Intra-chromosomal DNA double-strand break (DSB) joining events during immunoglobulin class switch recombination (CSR) require 53BP1. However, in BRCA1 mutant cells, 53BP1 blocks homologous recombination (HR) and promotes toxic NHEJ, resulting in genomic instability. Here, we identify the protein dimerization hub-DYNLL1-as an organizer of multimeric 53BP1 complexes. DYNLL1 binding stimulates 53BP1 oligomerization, and promotes 53BP1's recruitment to, and interaction with, DSB-associated chromatin. Consequently, DYNLL1 regulates 53BP1-dependent NHEJ: CSR is compromised upon deletion of Dynll1 or its transcriptional regulator Asciz, or by mutation of DYNLL1 binding motifs in 53BP1; furthermore, Brca1 mutant cells and tumours are rendered resistant to poly-ADP ribose polymerase (PARP) inhibitor treatments upon deletion of Dynll1 or Asciz. Thus, our results reveal a mechanism that regulates 53BP1dependent NHEJ and the therapeutic response of BRCAT-deficient cancers.

\footnotetext{
${ }^{1}$ Genome Integrity Laboratory, Wellcome Centre for Human Genetics, University of Oxford, Oxford OX3 7BN, UK. ${ }^{2}$ Division of Molecular Pathology, Oncode Institute, The Netherlands Cancer Institute, Amsterdam 1066 CX, The Netherlands. ${ }^{3}$ St. Vincent's Institute of Medical Research, Fitzroy, VIC 3065 , Australia. ${ }^{4}$ Genome Damage and Stability Centre, School of Life Sciences, University of Sussex, Falmer BN1 9RQ, UK. ${ }^{5}$ MRC Brain Network Dynamics Unit, Department of Pharmacology, University of Oxford, Oxford OX1 3TH, UK. ${ }^{6}$ Department of Oncology, Weatherall Institute of Molecular Medicine, University of Oxford, John Radcliffe Hospital, Oxford OX3 9DS, UK. ${ }^{7}$ CRUK/MRC Oxford Institute for Radiation Oncology, University of Oxford, Oxford OX3 7DQ, UK. ${ }^{8}$ Department of Medicine at St. Vincent's Hospital, Melbourne Medical School, The University of Melbourne, Fitzroy, VIC 3065, Australia. ${ }^{9}$ Institute of Animal Pathology, Vetsuisse Faculty, University of Bern, Bern 3012, Switzerland. These authors contributed equally to the work: Raquel Cuella-Martin, Marco Barazas, Rui Liu. Correspondence and requests for materials should be addressed to J.R.C. (email: rchapman@well.ox.ac.uk)
} 
T o counteract the potentially carcinogenic effects of DNA damage and mutation, cells employ a complex network of DNA repair pathways ${ }^{1}$. DNA double-strand breaks (DSBs) are among the most toxic of genomic lesions. They arise from a variety of endogenous and exogenous sources, including ionizing radiation (IR) treatments, replication fork collapse, and as programmed intermediates of antigen receptor gene rearrangements during variable, diversity and joining $(\mathrm{V}(\mathrm{D}) \mathrm{J})$ recombination and class-switch recombination (CSR $)^{2,3}$. DSBs are predominantly repaired by homologous recombination (HR) and nonhomologous end joining (NHEJ) pathways. HR is an essential DNA repair pathway owing to its utility in the repair of DSBs encountered during the S/G2-phases of proliferating cell populations ${ }^{4}$, and mutations affecting its fidelity are associated with tumourigenesis ${ }^{1}$. HR initiation involves the regulated nucleolytic resection of DSB termini in a $5^{\prime}-3^{\prime}$ direction, which is an important determinant of whether a given break is repaired by HR or NHEJ ${ }^{5}$. The resulting $3^{\prime}$-tailed ends are used to invade homologous sequences on the sister chromatid, which is used as a template for accurate repair. The availability of a sister chromatid in only replicated regions of the genome ensures that HR repair is largely restricted to S and G2 phase cells, while NHEJ predominates in $\mathrm{Gl}^{3}$. NHEJ instead involves the direct ligation of DSB ends, a mechanism utilized by developing and antigenstimulated lymphocytes to generate programmed deletions during the diversification of antigen receptor genes. However, inappropriate activity of the NHEJ pathway is also known to promote genomic rearrangements and translocations associated with the onset of cancer. Activation of the appropriate pathway for a given DSB type, cellular context, or genomic locus is therefore crucial for the maintenance of genome stability and avoidance of deleterious errors or mutations ${ }^{5}$.

TP53-binding protein 1 (53BP1) is a DSB responsive protein that mediates an important and specialised branch of the NHEJ pathway ${ }^{6}$. 53BP1 is rapidly recruited to DSB sites, where its interaction with modified nucleosomes in DSB-flanking chromatin functions to inhibit nucleolytic DNA end resection, favouring DNA joining by NHEJ. This involves binding to a combinatorial chromatin signature consisting of methylated histone $\mathrm{H} 4(\mathrm{H} 4 \mathrm{~K} 20 \mathrm{me} / 2)$ and ubiquitinated $\mathrm{H} 2 \mathrm{~A}$ (H2AK15Ub) within DSB-associated chromatin domains ${ }^{7,8}$, and the recruitment of downstream effector proteins such as RIF1 (RAP1 interacting factor 1 ), whose binding to $53 \mathrm{BP} 1$ is required for efficient DNA end-protection and NHEJ ${ }^{9-11}$. RIF1-53BP1 complexes are essential for immunoglobulin (Ig) CSR, an NHEJdriven deletional recombination reaction in activated $\mathrm{B}$ cells that mediates the replacement of excised default IgM-encoding constant $(C)$ gene segments of the Ig heavy locus (Igh), with downstream $C$ segments of different class and function (e.g., IgG, IgE and IgA). Mice deficient in 53BP1 or RIF1 are immunodeficient owing to CSR failure ${ }^{9,12-14}$, defects that manifest as a result of the aberrant hyper-resection of Igh DSBs that generate ssDNA intermediates non-amenable to $\mathrm{NHEJ}^{9,14,15}$. The 53BP1 pathway also plays an equivalent but pathological role in DNA end-joining at de-protected telomeres: telomeric DNA ends exposed upon disruption of the telomere capping complex Shelterin are predominantly repaired by 53BP1-dependent NHEJ, resulting in chromosome end fusions ${ }^{16}$. Accordingly, uncapped telomeric DNA ends are hyper-resected in 53bp1- and Rif1-deficient cells, resulting in a near complete suppression of telomere endjoining ${ }^{11,17}$.

53BP1-dependent NHEJ is also problematic in BRCA1-deficient cancers, where it mediates chromosomal rearrangements that drive oncogenesis ${ }^{18,19}$. In mice, germline 53bp1-deletion suppresses the embryonic lethality and mammary tumourigenesis associated with homozygous Brcal loss-of-function mutations, a rescue explained by reactivation of $\mathrm{HR}^{19}$. Conversely, 53BP1 pathway-associated DSB repair activities underlie the synthetic lethal effect of poly-ADP ribose polymerase (PARP) inhibitor (PARPi) treatments in BRCA1 mutation-associated cancers: genetic ablation of 53BP1 pathway components results in PARPi resistance in cellular and tumour models of BRCA1deficiency $9,20,21$.

Aside from DSB repair, distinct 53BP1 protein complexes regulate the activation of p53-dependent senescence programs triggered in response to p53-activating drug treatments and prolonged mitotic transit ${ }^{22-25}$. Here, 53BP1's C-terminal BRCT domains bridge p53's interaction with the deubiquitinating enzyme USP28, catalysing de-ubiquitination events that stimulate p53 binding to, and activation of, target gene promoters ${ }^{22}$.

While mechanistically distinct, both NHEJ and p53-regulatory functions require 53BP1 multimerization $22,26,27$, a function thought to rely entirely on its oligomerization domain (OD; encoded within amino acid residues (a.a.) 1231-1277) ${ }^{28}$. NHEJdeficits have been attributed to an inability to recruit 53BP1 to DSB-associated chromatin, as OD mutation/deletion blocks the retention of 53BP1 protein fragments at DNA damage sites, and also compromises their interaction with modified nucleosome core particles in vitro ${ }^{8,28,29}$. Perhaps unexpectedly, we and others recently reported that the recruitment of a full-length 53BP1 protein into IR induced foci (IRIF) was only modestly impacted by OD- deletion or mutation ${ }^{22,27}$. This prompted us to explore the molecular basis and function of OD-independent 53BP1 recruitment.

Here, we reveal that 53BP1 can interact with DNA damage sites independently of its OD, and this recruitment depends on interaction with the multifunctional homodimeric protein hub dynein light chain 1 (DYNLL1). We demonstrate that DYNLL1 binding promotes ordered 53BP1 oligomerization and is essential for efficient CSR and adaptive immunity in mice, while also contributing to 53BP1-dependent p53 regulation. In addition, we show DYNLL1-53BP1 interplay plays an essential role in mediating toxic NHEJ events in Brcal mutant cancer cells: deletion of DYNLL1 or its transcriptional regulator ATM substrate Chk2-interacting $\mathrm{Zn}^{2+}$ finger protein (ASCIZ; also known as ATMIN/ZNF822) is strongly selected for in BRCA1deficient tumour cells, and results in PARPi resistance in vitro and in vivo. Thus, our findings identify an important mechanism in which DYNLL1 directly regulates the fidelity of 53BP1dependent NHEJ.

\section{Results}

DYNLL1 is essential for OD-independent 53BP1 recruitment. The minimal domain requirements for 53BP1 DSB localization were defined in the context of a 53BP1 fragment encompassing a. a. 1220-171130,31. This fragment spans 53BP1's nuclear localization sequence (NLS), OD, tandem-tudor, and ubiquitindependent recruitment (UDR) domains, all of which were essential for its recruitment into IRIF 8,28 . We were therefore surprised that complete deletion of its $\mathrm{OD}$ domain $\left(53 \mathrm{BP} 1^{\mathrm{OD}} \Delta\right)$ or Ala mutation of four key residues for OD activity $(53 \mathrm{BP} 1 \mathrm{ODm})$ only negligibly impacted on 53BP1 IRIF frequencies, despite completely blocking recruitment in the context of truncated 53BP $1^{1220-1711}$ proteins (Fig. 1a). To map the sequences in 53BP1 that mediate OD-independent recruitment, IRIF localization patterns were examined across a panel of truncated 53BP $10 D m$ proteins in which 53BP1 sequences $\mathrm{N}$ - and C-terminal to a.a. 1220-1711 were restored (Fig. 1b and Supplementary Fig. 1a). We found that the addition of 113 residues $\mathrm{N}$-terminal to 53BP1 $1220-1711$ rescued 53BP1ODm recruitment into IRIF (Fig. 1b), implicating a.a. 1107-1219 in OD-independent 53BP1 recruitment. 
a

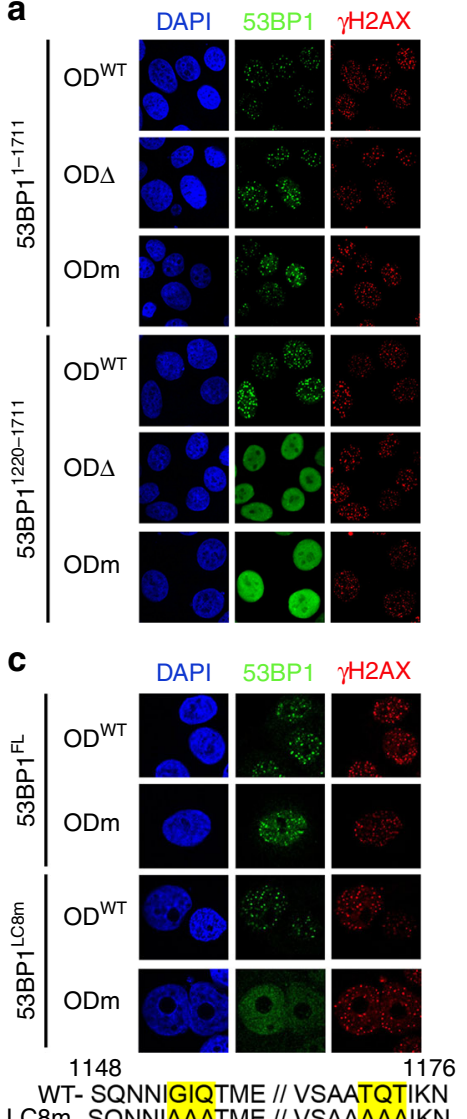

b

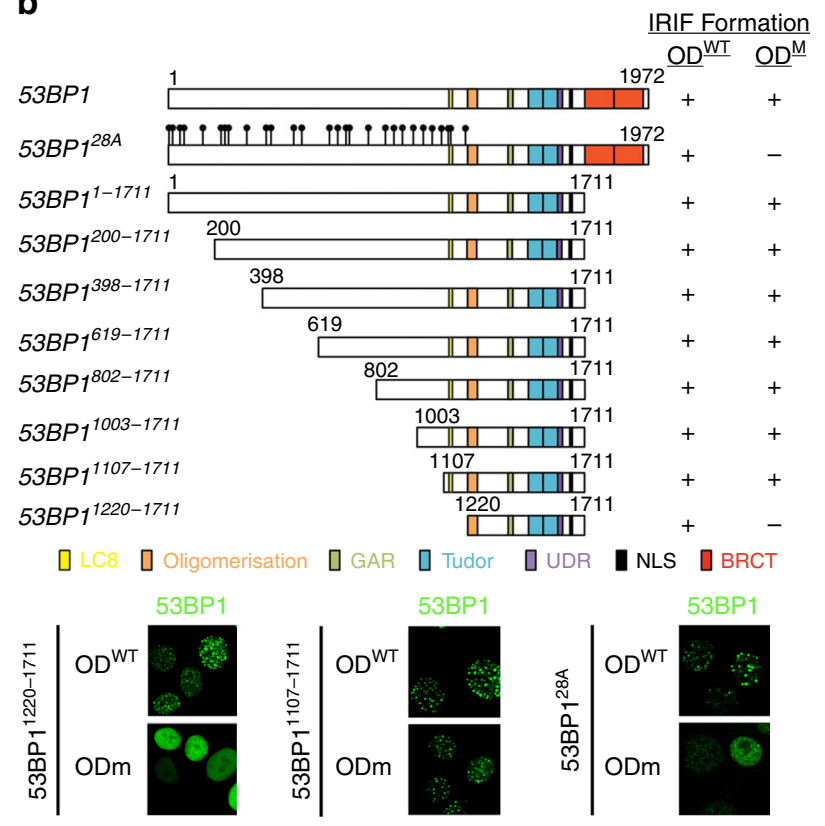

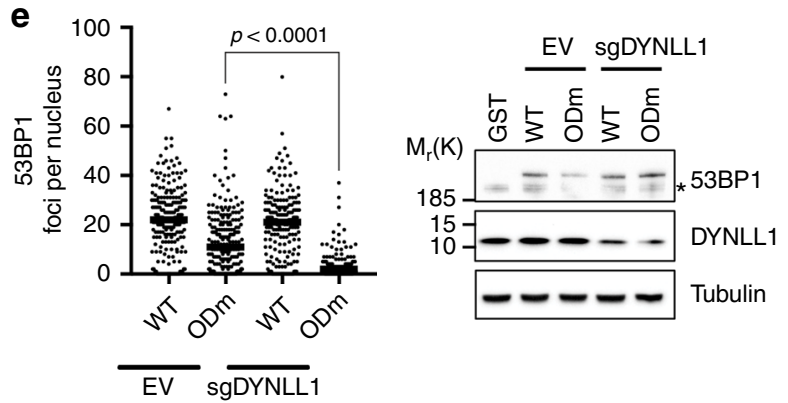
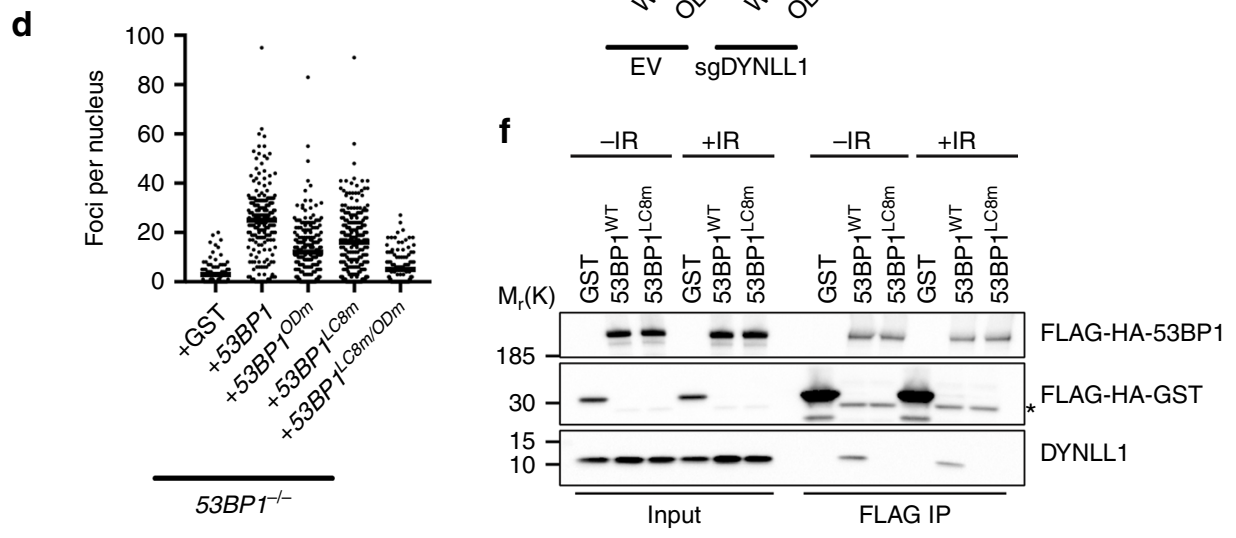

Fig. 1 DYNLL1 promotes OD-independent 53BP1 recruitment to DSB sites. a 53BP1\%- MCF-7 cells stably expressing the indicated 53BP1 transgenes were irradiated ( $5 \mathrm{~Gy}$ ), fixed $4 \mathrm{~h}$ later, and immunostained with anti-HA (53BP1) and anti- $\gamma \mathrm{H} 2 \mathrm{AX}$ antibodies. Data, representative of $n=3$ independent experiments. OD $\Delta$ indicates deletion of amino acids 1230-1270 and ODm indicates mutation of amino acids 1258-1261 to alanine. $\mathbf{b}$ IRIF forming capability of indicated 53BP1 constructs was determined in stable cell lines as in (a) $4 \mathrm{~h}$ following $5 \mathrm{~Gy}$ irradiation in a minimum of $n=2$ independent experiments. c As in (a-b), but with indicated WT and mutant 53BP1 constructs. Data, representative of $n=2$ independent experiments. Amino acids 1142-1181 of 53BP1 are depicted including 6 amino acids mutated to alanine in the LC8m allele. d Automated 53BP1 IRIF quantification of data in (c). Each point represents a single nucleus with $n \geq 65$ nuclei scored per condition. Median is indicated. Data, representative of $n=2$ independent experiments. e Left, quantification of 53BP1 IRIF $4 \mathrm{~h}$ after irradiation ( $5 \mathrm{~Gy}$ ). Significance determined by Mann-Whitney U Test. Right, immunoblot analysis of experimental cell lines. Each point represents a single nucleus with $n \geq 131$ nuclei scored per condition. Median is indicated. Data, representative of $n=2$ independent experiments. f FLAG-HA-53BP1 immuno-complexes were isolated from whole cell extracts prepared from untreated and irradiated (10 Gy, $1 \mathrm{~h}$ ) HEK $293 \mathrm{~T}$ cultures, $48 \mathrm{~h}$ following transfection with indicated control (GST) or 53BP1 expression plasmids. Representative data, $n=2$ independent experiments

Interestingly, alanine substitutions at $28 \mathrm{~N}$-terminal [Ser/Thr]Gln consensus ATM/ATR phosphorylation site motifs in the fulllength $53 \mathrm{BP} 1 \mathrm{ODm}$ protein recapitulated the effect of a.a. 1107-1219 deletion on IRIF recruitment (Fig. 1b), and suggested that some of these Ser-Gln or Thr-Gln motifs may be involved in the OD-independent 53BP1 recruitment to DSBs. We noted that
Thr-1171, one of the three Ser-Gln/Thr-Gln motifs in the region critical for OD-independent IRIF formation (a.a. 1107-1219), also serves as a binding site (consensus Thr-Gln-Thr) for the dynein light chain protein DYNLL1 (LC8) ${ }^{32}$. As DYNLL1 is known to function as a ubiquitous sequence-specific dimerization hub for a plethora of diverse proteins ${ }^{33,34}$, we considered a role 
for DYNLL1 in the OD-independent 53BP1 recruitment mechanism. 53BP1 a.a. 1107-1219 also contains a second DYNLL1-binding site ${ }^{32}$, and indeed, alanine substitution of the three anchor residues (GIQ and TQT, respectively) in each of the two DYNLL1-binding site motifs of 53BP1 (53BP1 $\left.1^{\mathrm{LC} 8 \mathrm{~m}}\right)$ completely blocked OD-independent 53BP1 IRIF formation (Fig. 1c-d). Moreover, we found that DYNLL1 colocalized with 53BP1 in nuclear foci and that this interaction was abolished by the 53BP $1^{\mathrm{LC} 8 \mathrm{~m}}$ mutations (Supplementary Fig. 1b). However, the $53 \mathrm{BP} 1^{\mathrm{LC} 8 \mathrm{~m}}$ mutation did not interfere with OD-mediated oligomerization in vitro (Supplementary Fig. 2a), or downstream RIF1 recruitment (Supplementary Fig. 2b, c). Thus, collectively, these data indicate that direct binding of DYNLL1 to the LC8binding motifs is critical for the OD-independent recruitment of 53BP1 to DSB sites.

To further explore DYNLL1's role in modulating 53BP1 functions, we used CRISPR-Cas9 to mutagenize the DYNLL1 gene in $53 \mathrm{BP}^{-/-} \mathrm{MCF}-7$ cell populations stably complemented with wild type (WT) $53 \mathrm{BP} 1$ or $53 \mathrm{BP} 1 \mathrm{ODm}$. DYNLL1-depletion in these populations (without selection for stable DYNLL1 knockout clones) caused a near complete block in 53BP1 $\mathrm{ODm}$ foci formation (Fig. 1e and Supplementary Fig. 2d, $p<0.0001$ ), and residual 53BP1 IRIF frequencies in these experiments correlated to cells of increased DYNLL1 nuclear staining intensity (Supplementary Fig. 2e). In contrast, DYNLL1-depletion did not affect the frequencies of WT 53BP1 IRIF when the OD was intact (Fig. 1e; $p=0.1363$ ). These experiments therefore suggested that DYNLL1-dependent binding to the LC8 motifs in $53 \mathrm{BP} 1$ promotes $53 \mathrm{BP} 1$ oligomerization, explaining the mechanism of OD-independent $53 \mathrm{BP} 1$ recruitment to IRIFs.

DYNLL1-53BP1 interactions are DNA damage- and cell cycleindependent. As indicated above, one of the two LC8 motifs (LC8 motif 2) in 53BP1 also comprises an ATM/ATR phosphorylation site (Thr-1171, consensus Thr-Gln) that is phosphorylated following IR-treatment ${ }^{35}$, and a second putative ATM phosphorylation site (Ser-1148) is positioned only 5 a.a. residues upstream of LC8 motif 1. Thus, we considered that 53BP1-DYNLL1 interactions might be DNA damage regulated. To test this, FlagHA tagged WT 53BP1 and 53BP1 $1^{\mathrm{LC} 8 \mathrm{~m}}$ mutant protein complexes were immunoprecipitated from lysates prepared from irradiated and untreated stable cell lines and analyzed for the presence of DYNLL1. In confirmation of a role for the 53BP1 LC8 motifs in mediating DYNLL1 interactions, DYNLL1 co-precipitated with wild type, but not LC8-motif mutant 53BP1 complexes (Fig. 1f). Notably, DYNLL1-53BP1 interactions were not significantly altered upon IR-treatments (Fig. 1f), discounting a role for Thr1171 phosphorylation in regulating DYNLL1-53BP1 interactions. In addition, mutation of Ser-1148, which would be expected to form the $\mathrm{N}$-terminal residue of the fully extended $\sim 8$-residue beta-strand interacting with the DYNLL1-binding groove based on known structures for DYNLL1-target complexes ${ }^{36}$, by itself had only a modest effect on DYNLL1 binding. Mutation of Thr1171, however, reduced DYNLL1-binding to a greater extent, which was further reduced in concert with the Ser-1148-Ala mutation, albeit not to the same extent as the $53 \mathrm{BP} 1^{\mathrm{LC} 8 \mathrm{~m}}$ interaction-blocking mutation in which the entire 3-residue anchor motifs are disabled (Supplementary Fig. 1c). Moreover, combined alanine substitutions at Thr-1171 and Ser-1148 resulted in the near total loss of OD-independent 53BP1 IRIF (Supplementary Fig. 1d). The most likely explanation for these results is that mutation of these two phosphorylation site motifs impacts the interaction with DYNLL1 by weakening its binding sites in 53BP1 rather than by impairing their phosphorylation state, reconciling the IRIF recruitment defect of the 53BP1ODm,28A mutant protein (Fig. 1b).
Next, we examined whether DYNLL1-53BP1 interactions might be regulated by cell cycle position. Cell synchronisation experiments confirmed DYNLL1 did not exhibit cell-cycledependent fluctuations in expression during interphase (Supplementary Fig. 3a), or mitosis (Supplementary Fig. 3b). To determine whether 53BP1 might be regulated by cell-cycle dependent modification of DYNLL1, or otherwise, 53BP1 IRIF were quantified in G1, S and G2 cell-cycle stage classified populations in WT and mutant complemented 53BP1 $1^{-/-}$MCF-7 stable cell lines (Supplementary Fig. 3c). Here, the 53BP1 LC8m mutation did not significantly impair foci formation at any cell cycle phase (Supplementary Fig. 3d). Likewise, 53BP1 ODm IRIF frequencies, that rely entirely on DYNLL1-interactions (see Fig. 1c-f), were equally reduced at all cell cycle phases (Supplementary Fig. 3d). Collectively, these experiments indicate DYNLL1-53BP1 interactions are likely to be constitutive, and suggested that DYNLL1 might represent an integral component of 53BP1 complexes.

53BP1-DYNLL1 interactions are required for class-switch recombination. We next examined the function of DYNLL1 and DYNLL1-53BP1 interactions during CSR, which relies on $53 \mathrm{BP} 1-$ mediated NHEJ ${ }^{12,13}$. DYNLL1 is essential for normal B cell development, and its deletion in the early B cell lineage using Mb1-Cre leads to dramatic losses in circulating and mature splenic B cell populations (Supplementary Fig. $4 \mathrm{a})^{37}$. We therefore used transgenic Cd23-Cre driver $\left(\mathrm{Cd} 23-\mathrm{Cr} \mathrm{e}^{\mathrm{Tg}}\right)$ to delete Dynll1 in mature B lymphocytes in Dynll1 ${ }^{F / F}$ mice, which supported the development of normal frequencies of mature splenic B cells in which DYNLL1 protein was efficiently depleted (Supplementary Fig. 4b, c). Cultured Dynll1-deleted B splenocytes upon stimulation with IL-4 and LPS showed a reduction in classswitching to IgG1, and IgG1 switching frequencies in $\mathrm{Cd} 23-\mathrm{Cre} \mathrm{Tg}^{\mathrm{Tg}}$ Dynll1 $^{F / F}$ cells were consistently reduced by $>50 \%$ relative to Cd23-Cre $e^{T g}$ controls in all cell populations that had undergone equivalent numbers of cell divisions (Fig. 2a, b). This was independently confirmed by $\mathrm{Cd} 23$-Cre-mediated deletion of the Asciz gene in mouse mature B cells, which encodes DYNLL1's transcriptional regulator ASCIZ (ATMIN) $)^{38,39}$ and resulted in greatly reduced DYNLL1 expression and an equivalent reduction in class-switching efficiency (Supplementary Fig. 4d, e), consistent with previous results in $\mathrm{B}$ cells from Cd19-Cre Asciz-mutant mice ${ }^{40}$. The fact that CSR defects in Dynll1- and Asciz-deleted B cells could not be explained by the defective expression of Igh switch region germ-line transcripts (Supplementary Fig. 4f), nor the magnitude of proliferation defects in Dynll1-deleted B cells (Fig. 2b), was consistent with a role for DYNLL1 and its regulator ASCIZ in the end joining phase of CSR.

To exclude the possibility that indirect, 53BP1-independent, consequences of DYNLL1-deletion could account for CSR defects in Dynll1-deficient B cells, we next monitored the effect of LC8 binding site mutations on 53BP1-dependent CSR. LPS/IL-4induced class-switching to IgG1 was analysed in stimulated $53 \mathrm{pp}^{-/-}$primary B splenocytes upon reconstitution with retroviruses that express wild type 53BP1, or the $53 \mathrm{BP} 1 \mathrm{ODm}$, $53 \mathrm{BP} 1^{\mathrm{LC} 8 \mathrm{~m}}$, and $53 \mathrm{BP} 1^{\mathrm{LC} 8 \mathrm{~m}} / \mathrm{ODm}$ mutant proteins, or as a negative control, the NHEJ-defective 53BP1 ${ }^{\text {L1619A }}$ UDR mutant ${ }^{8}$ (Fig. 2c; note that to avoid the inefficient packaging of large $53 B P 1$ inserts in retroviral particles, all rescue constructs express a truncated $53 \mathrm{BP} 1$ protein (a.a. 1-1711) that supports WT CSR frequencies $\left.{ }^{26}\right)$. As expected, 53BP1 $1^{1-1711}$ expression rescued class switching in $53 b \mathrm{p1}^{-/-} \mathrm{B}$ cell cultures, while the repair-deficient 53BP1 $1^{\mathrm{L} 1619 \mathrm{~A}}$ mutant could not (Fig. 2c, d). In contrast, $53 \mathrm{BP} 1^{\mathrm{LC} 8 \mathrm{~m}}$-reconstitution only restored CSR to $\sim 50 \%$ of $\mathrm{WT}$ (Fig. 2c, d), a defect consistent in magnitude to Dynll1-deficient B 
a
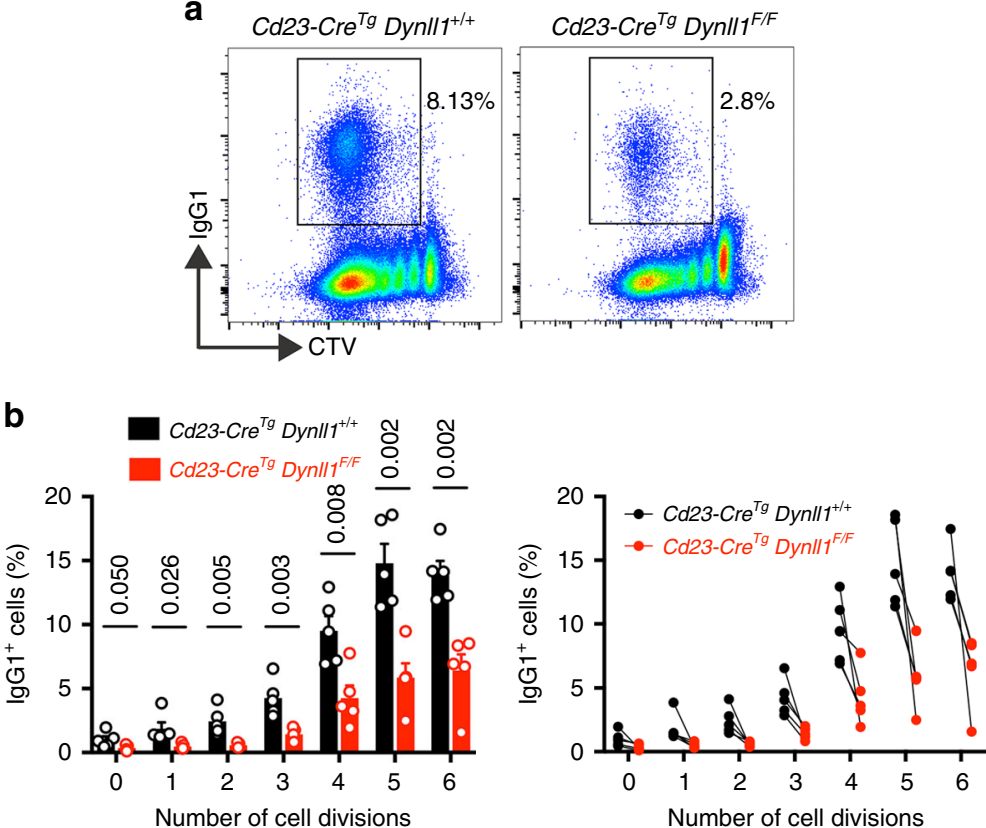

C

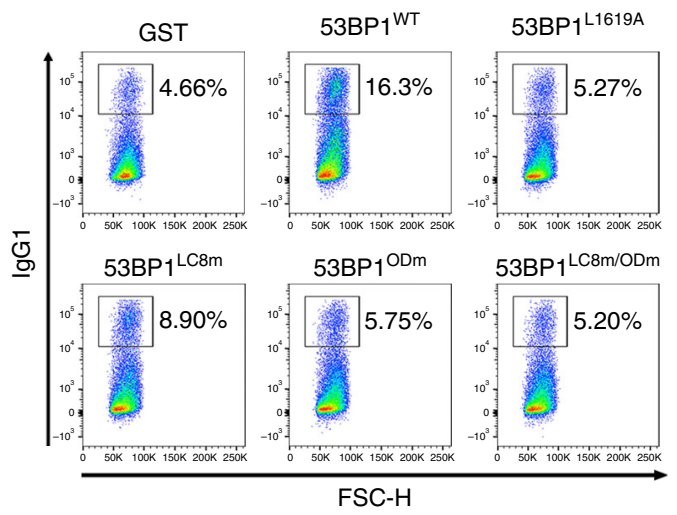

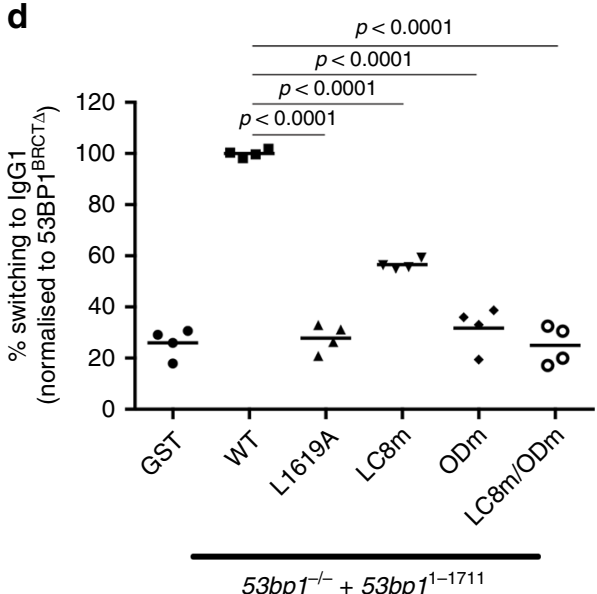

Fig. 2 53BP1-dependent class-switch recombination requires DYNLL1. a In vitro CSR to IgG1 in Dynl/1F/F Cd23-Cre ${ }^{T g}$ and control Cd23-Cre ${ }^{T g}$ mature B splenocytes, $96 \mathrm{~h}$ following stimulation with LPS and IL-4. Representative flow cytometric plots depict lgG1 positive fraction of cells, in cells co-stained with CellTrace Violet (CTV). b IgG1-positive ( $\left.\operatorname{lgG} 1^{+}\right)$B cells as a proportion of total B cells (\%) for each cell generation as determined by CTV staining and proliferation-associated dye dilution. Significance was determined by unpaired two-tailed student's t-test with Holm-Sidak correction for multiple comparisons. Data, $n=5$ mice, mean \pm SEM. c In vitro CSR to IgG1 in mature B splenocytes from 53bp 1 - mice, $96 \mathrm{~h}$ following stimulation with $\mathrm{LPS}$ and IL-4, and $72 \mathrm{~h}$ following retroviral complementation as indicated. Representative plots. d Quantification of (C). Bars represent mean, $n=4$ mice. Statistical analysis by unpaired two-tailed student's t-test

cells, confirming DYNLL1 mediates CSR via its regulation of 53BP1 complexes and their NHEJ activities. As expected ${ }^{26}$, $536 \mathrm{p}^{-1-\mathrm{B}}$ cells complemented with a mutant OD-domain allele $(53 \mathrm{BP} 1 \mathrm{ODm})$ failed to restore CSR to any significant degree (Fig. 2c, d). Thus, while DYNLL1 can mediate OD-independent 53BP1 DSB recruitment, OD-independent recruitment alone is unable to support NHEJ during CSR, confirming a cooperation between OD- and DYNLL1-dependent oligomerization in the assembly of NHEJ-competent 53BP1 oligomers.

DYNLL1 stimulates 53BP1 oligomerization to promote optimal chromatin interactions. To ascertain why DYNLL1mediated 53BP1 recruitment alone is insufficient for DNA repair, we investigated DYNLL1's contribution to 53BP1chromatin interaction dynamics at DSB sites. Stable 53BP1-/MCF-7 cell lines were established that expressed equivalent levels of an mC2 (mClover2)-tagged 53BP1 a.a. 1107-1711 fragment, comprising WT (mC2-f53BP1) or mutated versions of the OD $\left(\mathrm{mC} 2-\mathrm{f} 53 \mathrm{BP} 1^{\mathrm{ODm}}\right)$ or $\mathrm{LC} 8$ motifs $\left(\mathrm{mC} 2-\mathrm{f} 53 \mathrm{BP} 1^{\mathrm{LC}} \mathrm{m}\right)$. The mobility of each protein in IRIF was then calculated in fluorescent recovery after photobleaching (FRAP) experiments (Fig. 3a). Fluorescence recovery of $\mathrm{mC} 2-\mathrm{f} 53 \mathrm{BP} 1 \mathrm{ODm}$ was $>3$-fold faster than that of WT mC2-f53BP1 protein, as calculated from the half-time for recovery to maximum fluorescence: $t_{1 / 2}=47.3 \mathrm{~s} \pm$ 18.12 and $163.25 \mathrm{~s} \pm 46.9$, for $\mathrm{mC} 2-\mathrm{f} 53 \mathrm{BP} 1 \mathrm{ODm}$ and $\mathrm{mC} 2-\mathrm{f} 53 \mathrm{BP} 1$ proteins, respectively (Fig. 3b, c). Thus, DYNLL1-mediated 53BP1 oligomerization alone is insufficient for stable chromatin interaction at DSB sites. These results confirm canonical DYNLL1-independent oligomerization is primarily responsible for 53BP1 interactions with DSB-associated chromatin. To control for variation in fluorescence recovery endpoints between experimental repeats (Fig. 3d), we analyzed the rate of fluorescence recovery in a manner that, unlike $t_{1 / 2}$, was not related to the 

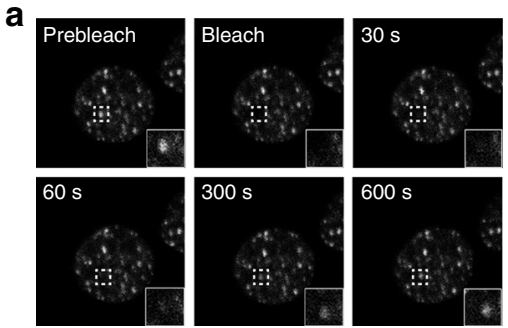

C

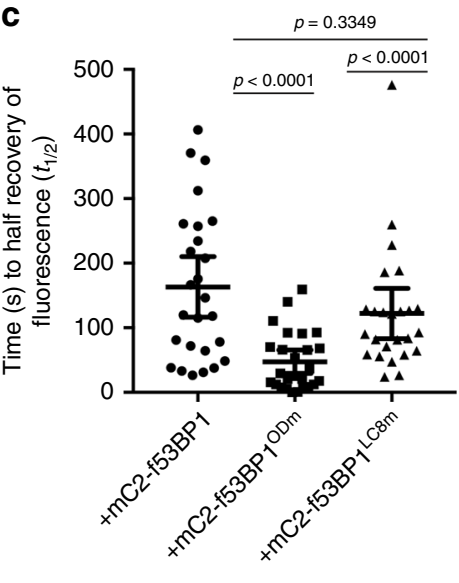

$53 B P 1^{-1-}$

\begin{tabular}{l|l}
\hline \multicolumn{1}{c|}{ Foci } & \multicolumn{1}{c}{$t_{1 / 2}(\mathrm{~s})$} \\
\hline $\mathrm{mC2}-f 53 \mathrm{BP} 1$ & $163.25 \pm 46.9$ \\
$\mathrm{mC2}^{-f 53 B P 1}{ }^{\text {ODm }}$ & $47.3 \pm 18.12$ \\
$\mathrm{mC2}^{-f 53 B P 1}{ }^{\mathrm{LC} 8 \mathrm{~m}}$ & $122.14 \pm 39.0$
\end{tabular}

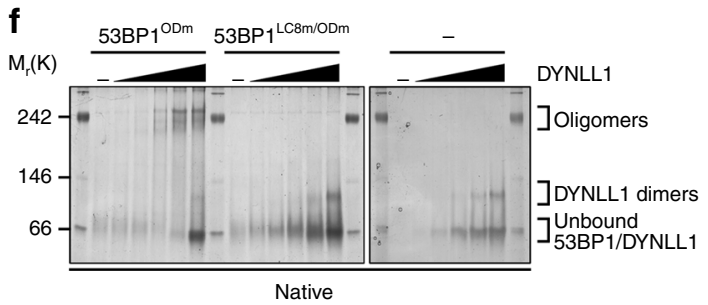

b
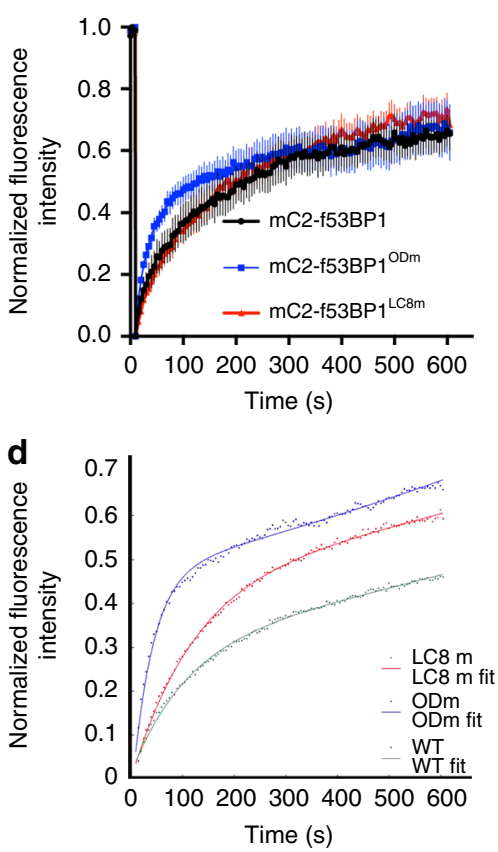

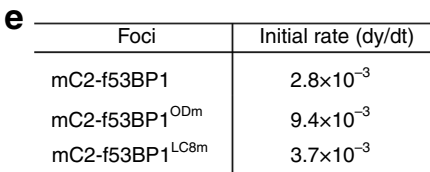

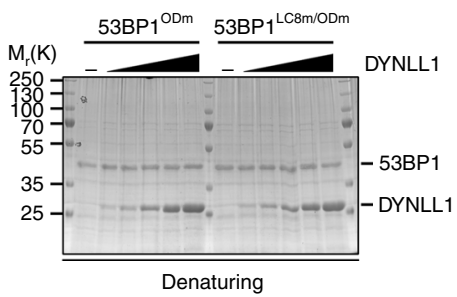

Fig. 3 Stable 53BP1-chromatin interactions require DYNLL1-dependent and -independent oligomerization modes. a Representative FRAP series. Example shows recovery of WT mC2-f53BP1. Bottom right corner shows magnified region of bleached area. All FRAP experiments were performed in 53BP1 $/-$ MCF7 cells stably expressing the indicated mC2-f53BP1 fragments. b Mean recovery from a single FRAP experiment in cells expressing mC2-f53BP1 ( $n=8$ ), $\mathrm{mC2}-\mathrm{f53BP1LC8m}(n=9)$, or mC2-f53BP1ODm $(n=10)$, mean \pm SEM. Data, representative of $n=3$ independent experiments. c Time for half-recovery of maximum final fluorescence for IRIF in individual cell expressing mClover2-WT $(n=26), \mathrm{mC2}-\mathrm{f} 53 \mathrm{BP} 1 \mathrm{LC} 8 \mathrm{~m}(n=26)$, or $\mathrm{mC2}-\mathrm{f} 53 \mathrm{BP} 1 \mathrm{ODm}(n=25)$ proteins. Mean $\pm \mathrm{Cl}(95 \%)$. P-values, Mann-Whitney $\mathrm{U}$ test. d Curves of best fit for three aggregated experiments. Data points represent the mean of $n \leq 25$ determinations. e Calculated initial rates (dy/dt) for the curves generated in (d). f Purified Smt3-His ${ }_{6}$-tagged DYNLL1 was incubated with Smt3-His ${ }_{6}-$ tagged 53BP1 $\mathrm{ODm}$ or 53BP1 $\mathrm{LC} 8 \mathrm{~m} / \mathrm{ODm}$ fragments (a.a. 1131-1292) as indicated. $1 \mu \mathrm{g}$ of 53BP1 was added to each reaction with increasing concentrations of DYNLL1. Molar ratios of DYNLL1:53BP1 from left to right; 1:4, 1:2, 1:1, 2:1, 4:1. Samples were fractionated by native (8\%) or denaturing (12\%) PAGE and stained with Coomassie Brilliant Blue. Data, representative of $n=2$ independent experiments

endpoint. Thus, we computationally modelled the fluorescence recovery data of three independent experiments (each comprising $n>8$ cells per genotype) to calculate the initial rate of fluorescence recovery immediately after photobleaching (Fig. 3d). Initial rate calculations provide a concentration-independent measurement of fluorescence recovery that is independent of fluorescence recovery endpoint. Consistent with $t_{1 / 2}$ measurements, the initial recovery rates of each protein reproduced the trends seen in Fig. $3 \mathrm{~b}$, c, with large, and moderate increases in the recovery rates of $\mathrm{mC} 2-\mathrm{f} 53 \mathrm{BP} 1 \mathrm{ODm}$ and $\mathrm{mC} 2-\mathrm{f} 53 \mathrm{BP} 1^{\mathrm{LC} 8 \mathrm{~m}}$ mutant proteins, respectively, relative to $\mathrm{WT} \mathrm{mC} 2-\mathrm{f53BP} 1$ (Fig. 3e). These data confirmed an important role for DYNLL1 in stabilizing ODdependent 53BP1-chromatin interactions.

To determine the mechanism by which DYNLL1 contributes to 53BP1 function, we next probed DYNLL1's ability to promote 53BP1 oligomerization in vitro. Thus, purified DYNLL1 was titrated into binding reactions that contained a recombinant monomeric 53BP1 protein fragment encoding the two LC8binding motifs and a mutated OD domain (a.a. 1131-1292). Reactions were then resolved by native PAGE to ascertain DYNLL1's ability to stimulate OD-independent 53BP1 oligomerization. In these experiments, increasing DYNLL1 to levels 
equimolar and above that of the 53BP1 fragment, stimulated its robust oligomerization as determined by a marked increase in higher molecular weight protein complexes (Fig. 3f). In confirmation of a role for the 53BP1 LC8 binding motifs in mediating the assembly of DYNLL1-bridged 53BP1 oligomers, higher molecular weight complexes did not form when both LC8binding motifs in the 53BP1 fragment were mutated, and these reactions were characterised by an increased presence of ligand-free DYNLL1 dimers (Fig. 3f). Taken together, our data indicate that optimal 53BP1 oligomerization is mediated via a cooperation between its OD-domain (Fig. 1a, b), and the binding of DYNLL1 to its upstream binding sites in 53BP1 (Fig. 3f). We therefore conclude that the intermolecular affinity afforded by DYNLL1-53BP1 interactions is sufficient to promote 53BP1 oligomerization, yet in isolation is insufficient to efficiently tether 53BP1 complexes to DSB-associated chromatin. We instead propose that DYNLL1 may provide order to large oligomeric 53BP1 complexes, a function required for optimal chromatin interactions and its associated DNA repair function.

PARPi hypersensitivity in Brca1-deficient cells requires 53BP1DYNLL1 cooperation. The fact that DYNLL1-mediated regulation of 53BP1 oligomerization enhances 53BP1-dependent CSR next prompted us to test its importance more broadly in 53BP1dependent NHEJ. The efficacy of PARPi in killing BRCA1-deficient cells is dependent on 53BP1, and its loss in BRCA1-deficient cell-lines and mice results in resistance to PARPi-induced cell death ${ }^{9,19-21}$. We therefore theorized that reduced efficiency of 53BP1-dependent NHEJ upon DYNLL1 depletion might likewise confer PARPi resistance to BRCA1-deficient cells. Using CRISPRCas9, we mutagenized the Dynll1 and Asciz genes in the KB1P-G3

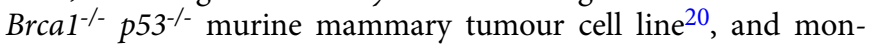
itored locus-specific indels within cell populations by deconvolution of complex Sanger sequencing traces derived from Cas9 cleavage site-spanning PCR amplicons using the Tracking of Indels by Decomposition (TIDE) algorithm ${ }^{41}$ (Supplementary Fig. 5a). To determine whether mutation of Dynll1 or Asciz provided a selective advantage in KB1P-G3 cells due to PARPi resistance, the change in percentage of edited alleles between the starting population and the surviving fraction was compared, before, and after, outgrowth in the presence or absence of the PARPi olaparib (Fig. 4a and Supplementary Fig. 5a). In all experiments in which the initial indel frequency at the Dynll1 and Asciz loci was below saturation $(<85 \%)$ in treatment naive cells, olaparib treatments selected for striking increases in indel frequency (Fig. 4b and Supplementary Fig. 5b). These effects were coupled to reductions in DYNLL1 protein levels when compared to untreated controls (Supplementary Fig. $5 \mathrm{c}$ ), collectively confirming that both proteins are required for the hypersensitivity of Brcal-deficient cells to PARPi. Cas9dependent mutagenesis of either gene in KB1P-G3 cells also conferred olaparib resistance in clonogenic survival experiments (Fig. 4c, d). Importantly, the Olaparib resistance in Asciz-mutated KB1P-G3 cells could be completely reversed by transgenemediated re-expression of exogenous DYNLL1 (Supplementary Fig. $6 a-c)$, confirming the importance of ASCIZ-mediated DYNLL1 transcription in the response of BRCA1-deficient cells to PARPi. However, neither selection for CRISPR-Cas9dependent Dynll1 or Asciz locus editing, nor reduced DYNLL1 expression, was observed when equivalent experiments per-

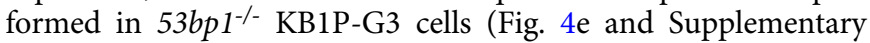
Fig. 5d, e), indicating that DYNLL1's role in mediating the PARPi sensitivity of Brcal-deficient cells occurs predominantly via its regulation of 53BP1 activities.
We next examined whether the ASCIZ-DYNLL1 axis was required for PARPi sensitivity in vivo. ASCIZ and DYNLL1 have previously been shown to have equivalent effects on the development and expansion of $M y c$-driven cancers $^{37,42}$, thus we monitored the growth of Cas9-expressing $\mathrm{BrCal}^{-/-} \mathrm{p53^{-/- }}$ transplanted mouse cancer organoids ${ }^{43}$ transduced with control or Asciz-targeting gRNA (Supplementary Fig. 5f, g). Olaparib treatment significantly delayed the onset of tumour growth in transplanted animals relative to an untreated cohort. However, PARPi-dependent inhibition of tumour growth was attenuated in Asciz-edited tumours (Fig. 4f), and resulted in decreased overall survival relative to control olaparib-treated cohorts (Fig. 4g). These data demonstrated that regulation of 53BP1 by the ASCIZDYNLL1 axis supports the efficacy of olaparib treatments in selectively killing Brcal-deficient cancer cells, confirming the importance of DYNLL1-53BP1 interactions in regulating 53BP1dependent DNA repair.

DYNLL1-binding sites contribute to 53BP1-dependent Nutlin3 sensitivity. In addition to its role in the DNA damage response, 53BP1 also contributes to p53-dependent transcriptional senescence programs and the corresponding cellular sensitivity to the treatments with MDM2 inhibitor Nutlin- $3^{22,44}$. Interestingly, the $53 \mathrm{BP} 1^{\mathrm{LC} 8 \mathrm{~m}}$ mutant, in contrast to wild type $53 \mathrm{BP} 1^{22}$, was compromised in their ability to restore the sensitivity of $53 \mathrm{BP}^{-/}$ MCF-7 to Nutlin-3 (Fig. 5a-c). This agrees with a model in which DYNLL1 is likely to enforce optimal 53BP1 oligomerization, and is consistent with our published findings in which efficient 53BP1 oligomerization was similarly essential for 53BP1-dependent regulation of $\mathrm{p} 53^{22}$.

\section{Discussion}

Taken together, our findings reveal DYNLL1 to be an integral component of oligomeric 53BP1 complexes through which it plays an important role in 53BP1-dependent NHEJ. We speculate that DYNLL1-binding to 53BP1 may provide order to 53BP1 oligomers within chromatin-binding domains, enhancing productive chromatin interactions that enhance effective resection inhibition and enable for efficient NHEJ. Deficiencies in either DYNLL1 or its binding sites in 53BP1, lead to substantial CSR defects. Given that 53BP1 is similarly important for resection inhibition and NHEJ during $\mathrm{V}(\mathrm{D}) \mathrm{J}$ recombination, a loss of which manifests in the defective development of $\mathrm{B}$ and $\mathrm{T}$ lymphocyte lineages $^{45-47}$, it is plausible that $53 \mathrm{BP} 1$-associated V(D)J recombination defects that accompany loss of DYNLL1-53BP1 interactions might contribute to the $\mathrm{B}$ cell lineage development defects recently reported in Dynll1-deficient mice ${ }^{37}$. We also found that DYNLL1 contributes to DNA damage-independent $53 \mathrm{BP} 1$ functions in the response to the MDM2 inhibitor Nutlin-3 (Fig. $5 \mathrm{a}-\mathrm{c}$ ). This agrees with a model in which DYNLL1 enforces optimal 53BP1 oligomerization, and is consistent with our published findings in which efficient 53BP1 oligomerization was similarly essential for 53BP1-dependent regulation of $\mathrm{p} 53^{22}$. Our results therefore suggest bivalent oligomerization modes are necessary to coordinate the assembly of 53BP1 molecules into ordered, functionally competent multimeric complexes, insights that will be relevant for understanding other macromolecular complexes organized by DYNLL1 (Fig. 6). In identifying DYNLL1 and ASCIZ as essential mediators of 53BP1-dependent PARPi sensitivity in Brcal-deficient cancer cells, our findings provide a molecular explanation for the identification of both proteins as PARPi resistance factors in BRCA1-deficient cells in recently published genetic screens ${ }^{48,49}$, and reveal a previously unanticipated mechanism in which 53BP1 misregulation could lead to PARPi resistance in the clinic. 
a

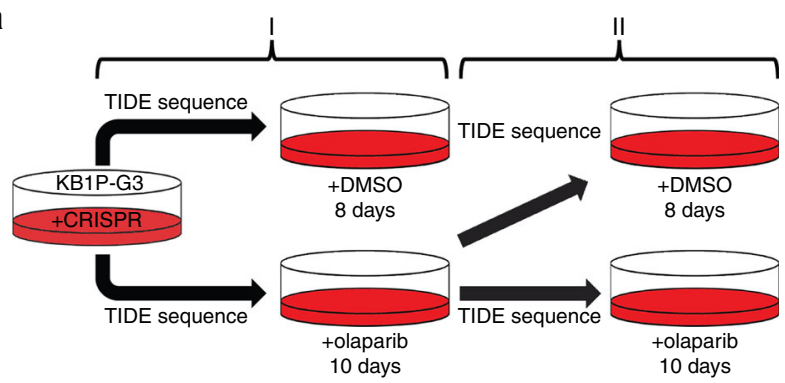

C

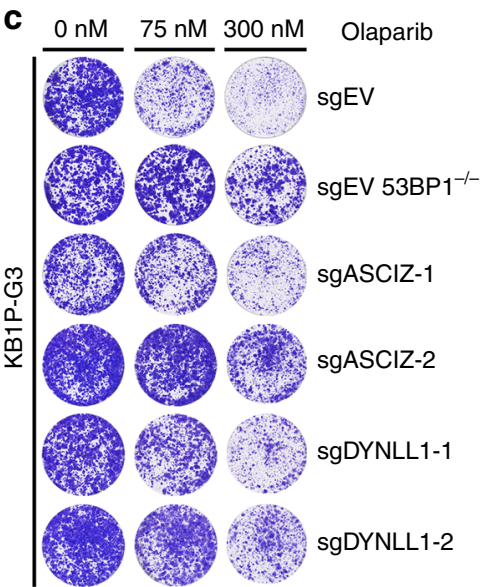

$\mathbf{f}$

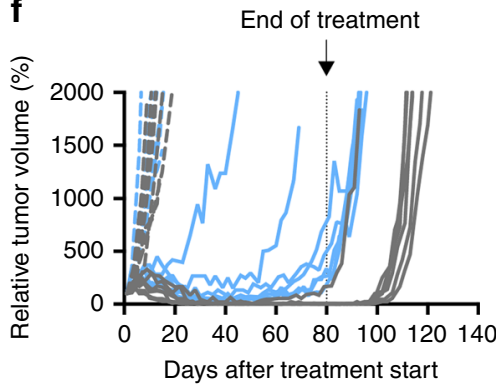

b

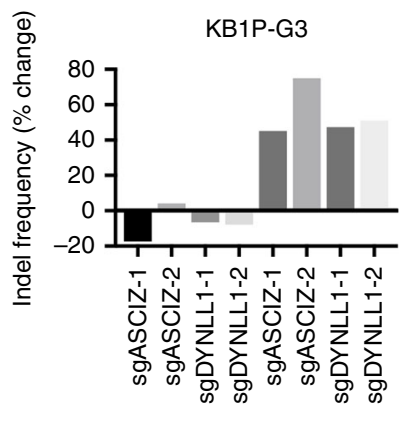

DMSO Olaparib

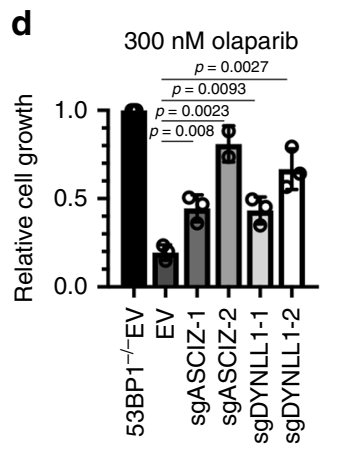

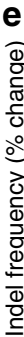

\) KB1P-G3 $53 b p 1^{-1-}$

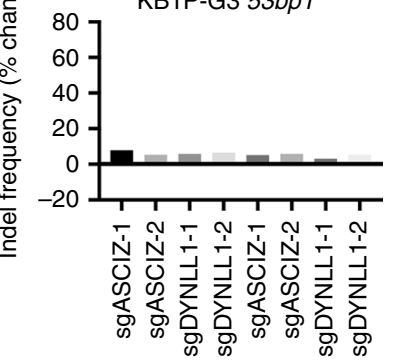

KB1P-G3

Olaparib

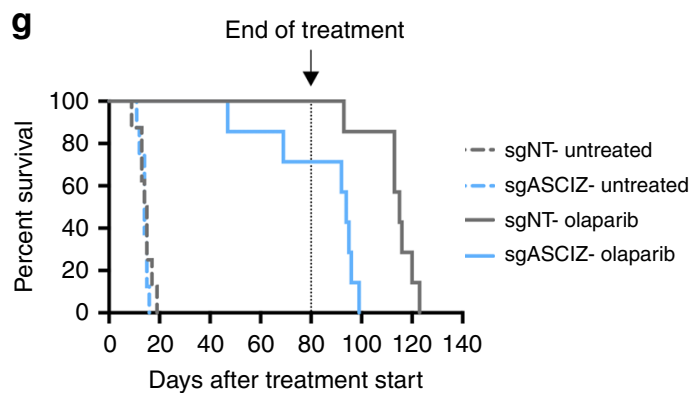

Fig. $4 \mathrm{ASCIZ}$ and DYNLL1 co-regulate 53BP1-dependent olaparib sensitivity in Brca1-deficient cells. a Schematic of experiments to determine olaparib resistance. $\mathbf{b}$ Change in the percent of edited alleles for the indicated target genes in KB1P-G3 cells after growth in DMSO or olaparib (75 nM). Data, representative of $n=3$ independent experiments. c Representative images from the second round of olaparib growth (II) in either DMSO or olaparib. d Quantification of growth in olaparib $(300 \mathrm{nM})$ after transduction with indicated guide RNAs. Quantification of $n=3$ independent experiments, each with three technical replicates. Mean \pm SD. Significance determined by unpaired two-tailed t test. e Change in the percent of edited alleles in $536 p 7^{\%}$ p $53^{-} \%$ KB1P-G3 cells after growth in DMSO or olaparib $(75 \mathrm{nM})$. Data, representative of $n=2$ independent experiments. $\mathbf{f}$ Relative tumour volume of transplanted organoids after treatment with olaparib. $n \geq 7$ animals per condition. $\mathbf{g}$ Kaplan-Meier plot indicating survival of organoid-transplanted animals. Significance between sgNT-olaparib and sgASCIZ-olaparib was determined by Log-rank Mantel-Cox test $(p=0.0018)$. $n \geq 7$ animals per condition

\section{Methods}

Cell lines and culture conditions. Cell lines used in this study are MCF-7 female human breast adenocarcinoma, RPE-1 female human retinal pigment epithelial, human female embryonic kidney (HEK) 293T cells (including the BOSC23 derivative cell line), and $\mathrm{KB1P}-\mathrm{G} 3 \mathrm{Brcal}^{-/} \mathrm{p} 53^{--}$cells which were isolated from a female mouse mammary tumour. All cell lines were checked for mycoplasma contamination. KB1P-G3 and the KB1P-G3 53bp $1^{-/-}$derivative were both kindly provided by Sven Rottenberg (NKI, Netherlands and University of Bern). All cell lines were cultured in DMEM-high glucose (D6546, Sigma-Aldrich) supplemented with $10 \%$ FBS, Pen-Strep, and $2 \mathrm{mM} \mathrm{L}$-glutamine. Cultures were grown at $37^{\circ} \mathrm{C}$ with $5 \% \mathrm{CO}_{2}$. KB1-P3 (G3) and all derivative cells lines were additionally grown in 3\% oxygen. Primary B cells were isolated from red blood cell-lysed single-cell suspensions of 53b $\mathrm{pl}^{-/-} \mathrm{C} 57 \mathrm{BL} / 6 \mathrm{~J}$ mouse spleens and cultured in RPMI supplemented with $10 \%$ FCS, $100 \mathrm{U} / \mathrm{ml}$ penicillin, $100 \mathrm{ng} / \mathrm{ml}$ streptomycin, $2 \mathrm{mM}$ L-glutamine, $1 \mathrm{x}$ MEM nonessential amino acids, $1 \mathrm{mM}$ sodium pyruvate and $50 \mu \mathrm{M} \beta$-mercaptoethanol.

Mouse models. Mice harbouring conditional Dynll1 or Asciz alleles (MGI ID: 5755433 and 5463697, respectively) were intercrossed with Cd23-Cre transgenic mice (MGI ID: 3803652) to generate Cd23-Cre ${ }^{T g} \mathrm{Dynll}^{\mathrm{F} / F}$ and Cd23-Cre ${ }^{T g} \mathrm{Asciz}{ }^{\mathrm{F} / F}$ experimental and $\mathrm{Cd} 23-\mathrm{Cr} e^{T g}$ control animals. Mouse breeding and tissue collection was performed according to the Australian Code for the Care and Use of Animals for Scientific Purposes, 8th Edition (2013), and approved by the St. Vincent's Hospital Melbourne Animal Ethics Committee, approval number 002/17. 53bp $1^{-1-}$ C57BL/6J mouse breeding and tissue collection was carried out according to the Animals (Scientific Procedures) Act (ASPA) under a Home Office project license to JRC.

Gene editing and complementation. CRISPR-Cas9 editing was carried out with gene-specific gRNAs integrated into a version of lentiCRISPR v2 (Addgene \#52961) modified for blasticidin (lentiCRISPR-Bsr) resistance. Lentivirus was generated by co-transfection of lentiCRISPR-Bsr, pHDM-tatlb, pHDM-G, pRC/CMV-revlb, and pHDM-Hgpm2 into HEK 293T cells using $1.29 \mu \mathrm{g}$ polyethylenimine per $1 \mu \mathrm{g}$ DNA in Opti-MEM (Thermo Fisher, 31985062). Viral supernatants were harvested at 48 and $72 \mathrm{~h}$ and passed through a $0.45-\mu \mathrm{m}$ syringe filter. Two rounds of viral transduction were carried out on target cell populations in the presence of $4 \mu \mathrm{g} / \mathrm{ml}$ polybrene before selection in $10 \mu \mathrm{g} / \mathrm{ml}$ blasticidin. Once all cells in a nontransduced control population had died, the CRISPR population was removed 
a

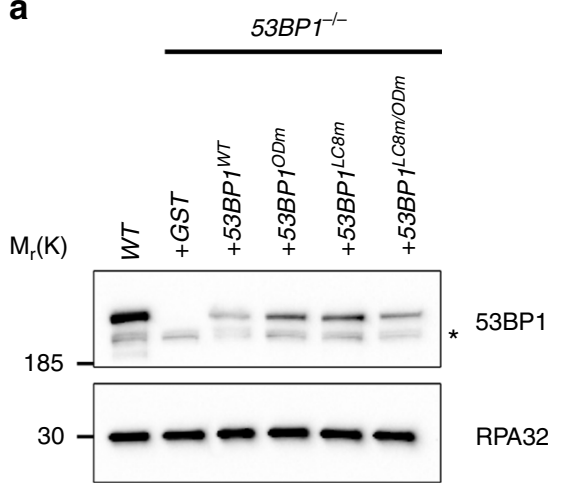

C

b

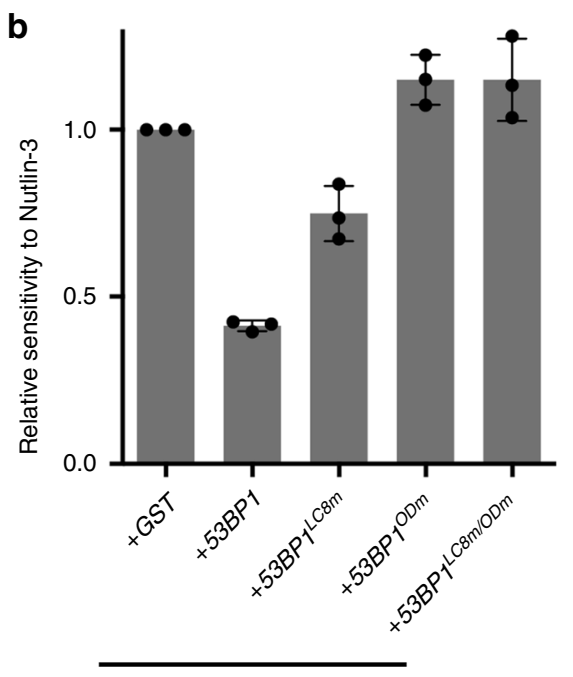

$53 B P 1^{-1-}$

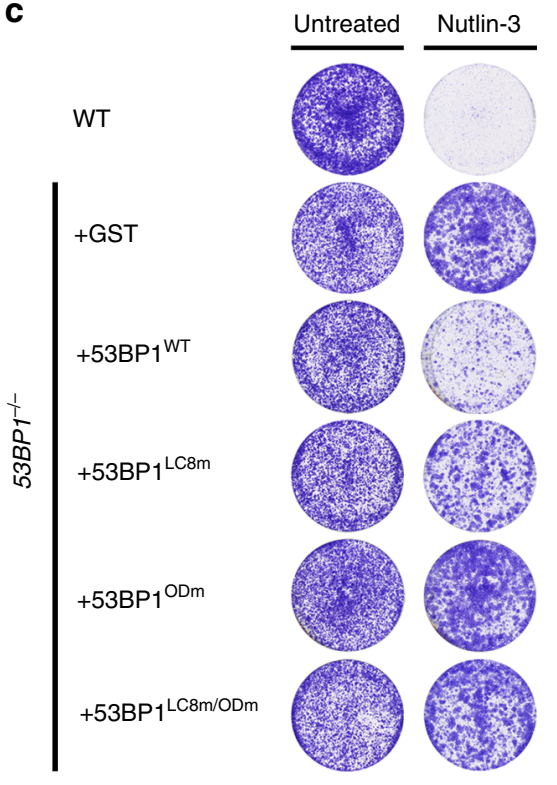

Fig. 5 DYNLL1 is required for 53BP1-dependent p53 responses to Nutlin-3. a Immunoblot analysis of the MCF-7 cell lines used in (a) with anti-53BP1 or anti-RPA32 antibodies prior to N3 treatment. b Quantification of $n=3$ independent experiments represented in (a), each performed in triplicate. Mean \pm SD. c Indicated parental or stably complemented 53BPT-/- MCF-7 cell lines were incubated in the presence (11 days) or absence (7 days) of Nutlin-3 (4 $\mu$ M)

from blasticidin selection. Stable transgene expression was similarly achieved by two rounds of lentiviral transduction with viral supernatants generated using the same procedure.

Immunofluorescence. For analysis of fixed samples, cells were seeded $\left(1.5 \times 10^{5}\right)$ on glass coverslips $(13 \mathrm{~mm})$ in six-well plates 2 days before irradiation. IRIF formation was induced with $\gamma$-irradiation $(5 \mathrm{~Gy})$ and cells were fixed in $2 \%$ paraformaldehyde after $4 \mathrm{~h}$ recovery. Fixed cells were rinsed with PBS and then permeabilized in a $0.2 \%$ Triton X-100 PBS solution. After 15 min of blocking (3\% BSA, $0.1 \%$ Triton $\mathrm{X}-100$ in PBS) coverslips were incubated $1 \mathrm{~h}$ in a humidity chamber with primary antibodies diluted in blocking solution. The following primary antibodies were utilized at the indicated concentrations: mouse anti-HA (1:200, HA.11 Biolegend), rabbit anti- $\gamma$-H2AX (1:500, Epitomics), rabbit antiCyclin A (1:200, Santa Cruz sc-751), rabbit anti-DYNLL1 (1:250, AbCam Ab51603). The coverslips were rinsed three times with PBS containing $0.1 \%$ TX100 and then incubated $1 \mathrm{~h}$ in a humidity chamber with secondary antibody. The following secondary antibodies were utilized at the indicated concentrations: goat anti-mouse Alexa Fluor 488 (1:500, Invitrogen A-11001), goat anti-rabbit Alexa Fluor 568 (1:500, Invitrogen A-11011). Coverslips were then washed three times in PBS containing $0.1 \%$ TX-100, once in PBS, and mounted on glass microscope slides using ProLong ${ }^{\oplus}$ Gold antifade reagent with DAPI (Life Technologies, P36935).

In experiments involving EdU incorporation, cultures were pulsed with $40 \mu \mathrm{M}$ EdU for $10 \mathrm{~min}$ immediately preceding irradiation. After fixation, EdU was labelled using the Click-iT ${ }^{\mathrm{T}} \mathrm{EdU}$ Alexa Fluor 647 Imaging Kit (Thermo Fisher, C10340) according to the manufacturer's protocol. This was done immediately prior to antibody staining as described above.

Images for the initial mapping of the LC8 domain within the 53BP1 N-terminus (Fig. 1) were acquired on a Zeiss LSM510 META confocal imaging system (Fig. 1). RIF1 IRIF (Supplementary Fig. 2b, 2c) were analyzed on a Leica SP8 SMD X confocal microscope. All other fixed IRIF images were acquired on an Olympus Epi-fluorescence MMI CellEctor widefield microscope. Quantitative analysis of IRIF number and staining intensity was performed using Cell Profiler (Broad Institute). All image visualization and processing was done using Fiji.
Olaparib sensitivity assays. KB1P-G3 and KB1P-G3 53bp $1^{-/-}$cells were subjected to two rounds of lentiviral transduction with viral supernatant generated using lentiCRISPR-Bsr. After selection in blasticidin $(10 \mu \mathrm{g} / \mathrm{ml})$, samples were collected to isolate genomic DNA (gDNA) immediately prior to seeding for olaparib sensitivity. Blasticidin-resistant populations were seeded in six-well plates at a density of $10^{4}$ cells per well $\left(5 \times 10^{3}\right.$ for the $\mathrm{KB} 1 \mathrm{P}-\mathrm{G} 353 \mathrm{bp1^{-/- }}$ derivative $)$ in the presence of olaparib or DMSO and grown at $37^{\circ} \mathrm{C}\left(5 \% \mathrm{CO}_{2}\right.$ and $\left.3 \% \mathrm{O}_{2}\right)$. Medium was refreshed at 4 days and 8 days. After 10 days outgrowth, cultures were expanded in fresh medium for 1 week in $6 \mathrm{~cm}$ dishes before harvesting gDNA. PCR fragments encompassing the CRISPR cut site were amplified from gDNA using Q5 polymerase (NEB, M0491), sequenced (GATC Biotech), and analyzed by Tracking of Indels by Decomposition (TIDE: https://tide.deskgen.com/). Surviving cells were collected and three replicates were plated in DMSO and three in olaparib for viability analysis. DMSO and olaparib-treated cells were stained with crystal violet $\left(0.5 \%(\mathrm{w} / \mathrm{v})\right.$ crystal violet in $25 \%$ methanol) after 8 days and 10 days growth $\left(37^{\circ} \mathrm{C}\right.$, $5 \% \mathrm{CO}_{2}$ and $3 \% \mathrm{O}_{2}$ ), respectively. Crystal violet stained cells were dissolved in a $10 \%(\mathrm{v} / \mathrm{v})$ acetic acid solution a minimum of $24 \mathrm{~h}$ after staining and the $\mathrm{OD}_{595}$ was measured as a quantitative metric of relative growth.

Nutlin-3 sensitivity assay. Cells were seeded in triplicate at a density of $1.25 \times 10^{4}$ per well in a six-well plate in the presence (11 days growth) or absence ( 7 days growth) of Nutlin-3 ( $4 \mu \mathrm{M}$, Cayman Chemicals, added $16 \mathrm{~h}$ after seeding). Cells were stained with crystal violet and quantified as described for olaparib sensitivity assays. These experiments were performed in triplicate.

Protein extraction and western blotting. Protein extracts were prepared in ice cold benzonase cell lysis buffer $(40 \mathrm{mM} \mathrm{NaCl}, 25 \mathrm{mM}$ Tris $\mathrm{pH} 8.0,0.05 \%$ SDS, $2 \mathrm{mM} \mathrm{MgCl}_{2}, 10 \mathrm{U} / \mathrm{ml}$ benzonase, and cOmplete ${ }^{\mathrm{Tw}}$ protease inhibitor cocktail (Roche, 04693159001). Briefly, cells were harvested and washed once in ice cold PBS before resuspending in lysis buffer. After 10 min incubation on ice, protein concentration was calculated by Bradford (Bio-Rad, 500-0006) assay with reference 

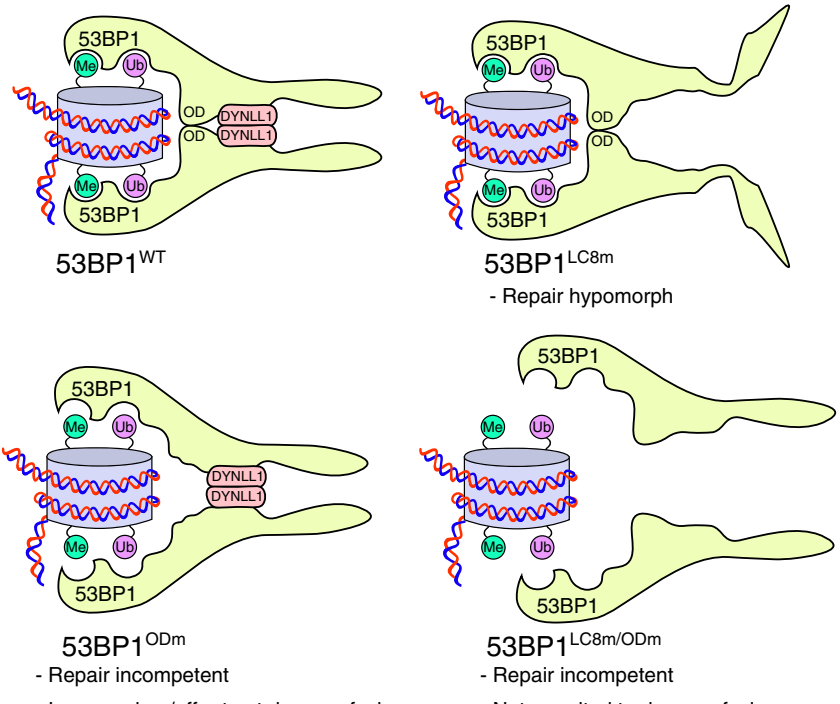

- Increased on/off rate at damage foci

Fig. 6 DYNLL1 and the OD mediate a bivalent mode of 53BP1 oligomerization. 53BP1 localisation and function is dependent upon bivalent OD- and DYNLL1-mediated modes of oligomerization (top, left). ODmediated oligomerization alone is sufficient for chromatin binding but results in severe DSB repair defects (top, right). DYNLL1-mediated oligomerization is also sufficient to support recruitment of 53BP1 to damage sites, but does not support DNA repair (bottom, left). Loss of both OD and DYNLL1-mediated modes of oligomerization eliminates 53BP1 recruitment to DNA damage sites and all associated repair functions (bottom, right)

to a standard curve generated with BSA. Extracts were mixed with $3 \mathrm{X}$ Laemmli buffer and heated at $95^{\circ} \mathrm{C}$ for $5 \mathrm{~min}$.

Extracts were fractionated on pre-cast NuPAGE ${ }^{\mathrm{Tm}} 4-12 \% 1.0 \mathrm{~mm}$ Bis-Tris polyacrylamide gels (Life Technologies, NP0322) and transferred to nitrocellulose membranes. After blocking with $5 \%$ milk and washing in PBST, membranes were incubated overnight in primary antibody. Primary antibodies used in this study are: anti-53BP1 (Novus Biological, NB100-304, 1:2500), anti-DYNLL1 (Abcam, ab51603, 1:1000), anti- $\alpha$-tubulin (Sigma-Aldrich, TAT-1, 1:10000), anti-Cyclin A (Santa Cruz, sc53227, 1:1000), anti-HA (BioLegend, 501901, 1:2000), and antiRPA32 (Abcam, ab16855, 1:1000), anti- $\beta$-actin (Sigma-Aldrich, A1978, 1:2000). Membranes were then probed with HRP-conjugated goat anti-mouse (Thermo Fisher, 62-6520) or goat anti-rabbit (Thermo Fisher, 65-6120) secondary antibodies for $1 \mathrm{~h}(1: 20000)$. The membranes were then developed using Clarity ${ }^{\mathrm{m}}$ Western ECL Substrate (Bio-Rad, 170-5061) and visualized on a Gel Doc ${ }^{\mathrm{mm}}$ XR System (Bio-Rad).

Class switch recombination. B cells were purified from red blood cell-lysed single-

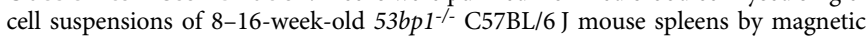
negative selection using a B Cell Isolation Kit (Miltenyi Biotec, 130-090-862). No controls were employed for gender and randomization methods were not used to allocate animals to control and experimental groups. B cells $\left(7.5 \times 10^{5}\right.$ per well in a six-well plate) were cultured in RPMI supplemented with $10 \%$ FCS, $100 \mathrm{U} / \mathrm{ml}$ penicillin, $100 \mathrm{ng} / \mathrm{ml}$ streptomycin, $2 \mathrm{mM}$ L-glutamine, 1x MEM nonessential amino acids, $1 \mathrm{mM}$ sodium pyruvate and $50 \mu \mathrm{M} \beta$-mercaptoethanol. B cells were stimulated with $5 \mu \mathrm{g} / \mathrm{ml}$ LPS (Sigma, L7770-1MG) and $10 \mathrm{ng} / \mathrm{ml}$ mouse recombinant IL-4 (Peprotech, 214-14-20). Cultures were grown at $37^{\circ} \mathrm{C}$ with $5 \% \mathrm{CO}_{2}$ under ambient oxygen conditions.

Retroviral supernatants were collected $48 \mathrm{~h}$ after co-transfection of BOSC23 cells with $7 \mu \mathrm{g}$ pCL-Eco and $7 \mu \mathrm{g}$ pMX-IRES-GFP-derived plasmids (both kindly provided by A. Nussenzweig) containing cloned transgene inserts in the presence of FuGENE 6 (Promega, E2691). Viral supernatants were passed through a $0.45 \mu \mathrm{m}$ syringe filter. One round of viral transduction was carried out on B cells $24 \mathrm{~h}$ after stimulation in the presence of $2.5 \mu \mathrm{g} / \mathrm{ml}$ polybrene, $20 \mathrm{mM}$ HEPES, $5 \mu \mathrm{g} / \mathrm{ml}$ LPS and $10 \mathrm{ng} / \mathrm{ml}$ mouse recombinant IL-4. B cells underwent spinoculation at $850 \times g$ for $90 \mathrm{~min}$ at $30^{\circ} \mathrm{C}$. After a rest period of 4 to $6 \mathrm{~h}$, the viral supernatants were removed, and supplemented RPMI was added to the cells.

Three days after transduction, infected B cells were analysed for the percentage of GFP and IgG1 double-positive cells using a FACSCanto (Becton Dickinson); analysis was performed using FlowJo v10. Cells were resuspended in PBS with $2 \%$ BSA and $0.025 \%$ sodium azide, blocked with Mouse BD Fc Block ${ }^{\text {Th }}$ (1:500, BD Pharmingen 553141), and immunostained with biotinylated rat anti-mouse IgG1 (1:100, BD Pharmingen 553441), and Streptavidin APC (1:500, eBioscience 17-

4317-82). Live/dead cells were discriminated after staining with Zombie Aqua viability dye (1:200, BioLegend 423102).

For CSR analyses of Cd23-Cre ${ }^{T g}$ Dynll1 ${ }^{F / F}$ and $C d 23-C r e^{T g} A s c i z^{F / F}$ mice, experiments were performed as above except that purified $\mathrm{B}$ cells were activated with $10 \mu \mathrm{g} / \mathrm{ml}$ LPS (Invivogen, tlrl-3pelps) and 1:100-diluted conditioned murine IL-4 supernatant (kind gift of Andreas Strasser, Walter and Eliza Hall Institute, Melbourne) for 4 days, and then stained with CellTrace Violet (Thermo Fisher Scientific, C34557) and rat anti-mouse IgG1-APC (BD Pharmingen, 550874).

To analyse the transcription of $I g h$ germline transcripts, $3 \times 10^{6}$ MACS-purified B cells from $\mathrm{Cd} 23-\mathrm{Cr} e^{T g}, \mathrm{Cd} 23-\mathrm{Cr} e^{T g} \mathrm{Asciz}{ }^{\mathrm{F} / F}$, or $\mathrm{Cd} 23-\mathrm{Cr} e^{T g} \mathrm{Dynll1}^{\mathrm{F} / \mathrm{F}}$ mice were cultured with LPS $(15 \mu \mathrm{g} / \mathrm{mL})$ and IL-4 (1/100) for 0 to 3 days. After treatment, cells were washed once with ice-cold PBS and cell pellets were snap frozen on dry ice and stored at $-80^{\circ} \mathrm{C}$ before RNA extraction. Total RNA was extracted by using ISOLATE II RNA Micro Kit (Bioline BIO-52075) following the manufacturer's protocol. $300 \mathrm{ng}$ purified RNA was subjected to reverse transcription with oligo (dT) and random primers using the SuperScript ${ }^{\oplus}$ III First-Strand Synthesis System (Invitrogen $\left.^{\text {TM }} 18080-051\right)$. qPCR reagents were mixed as follows: $4 \mu \mathrm{l}$ cDNA (6 ng), $0.5 \mu$ Primer mix (300 nM each), $5 \mu$ SYBR $^{\text {mo }}$ Select Master Mix (Applied Biosystems $^{\text {Tx }}$ 4472908), $0.5 \mu \mathrm{l}$ RNAase/DNAase free water. qPCR conditions were set as follows: 1 cycle $\left(10 \mathrm{~min}\right.$ at $\left.95^{\circ} \mathrm{C}\right), 45$ cycles $\left(15 \mathrm{~s}\right.$ at $\left.95^{\circ} \mathrm{C}\right), 60 \mathrm{~s}$ at $60^{\circ} \mathrm{C}$.

After each PCR run, a disassociation curve program was added to validate the primer specificity. The standard curve method of comparative quantification was employed to quantify the results ${ }^{50}$. Results for the $I \gamma 1-C \gamma 1$ germline transcript were normalized to the $C d 79 b$ input control, and expressed as percentage of maximum expression. Primer sequences were the same as previously used ${ }^{51}$ : Cd79b_forward (5'-TGTTGGAATCTGCAAATGGA-3), Cd79b reverse (5'-TAGGCTTT GGGTGATCCTTG-3), I $\gamma 1-C \gamma 1$ _forw (5'-TCGAGAAGCCTGAGGAATGTG-3), I $\gamma 1-C \gamma 1 \_r e v(5 '-G G A T C C A G A G T T C C A G G T C A C T-3)$.

Immunoprecipitation. One day before transfection, $4.5 \times 10^{6}$ HEK $293 \mathrm{~T}$ cells were seeded in antibiotic-free DMEM in a $10 \mathrm{~cm}$ plate for each condition. $24 \mathrm{~h}$ after plating, cultures were transfected with $10 \mu \mathrm{g}$ 53BP1 pLV-EF1a-FLAG-HA Puro or 53BP1 ${ }^{\text {LC8m }}$ pLV-EF1a-FLAG-HA Puro using polyethylenimine. $24 \mathrm{~h}$ after transfection, each culture was trypsinized and re-plated in $2 \times 10 \mathrm{~cm}$ plates. After $24 \mathrm{~h}$, one plate was X-ray irradiated (10 Gy) and both (irradiated and nonirradiated control) were harvested $1 \mathrm{~h}$ after irradiation. Cells were lysed in benzonase-containing lysis buffer (20 mM HEPES [pH 7.9], $40 \mathrm{mM} \mathrm{KCl,} 2 \mathrm{mM}$ $\mathrm{MgCl}_{2}, 10 \%$ glycerol, $0.5 \% \mathrm{NP} 40,0.05 \%$ [v/v] phosphatase inhibitor cocktails 2 and 3 (Sigma-Aldrich), 1X protease inhibitor (Roche), and benzonase [50 U/ml]) on ice for $10 \mathrm{~min}$. $\mathrm{KCl}$ was adjusted to a final concentration of $450 \mathrm{mM}$ and rotated at $4{ }^{\circ} \mathrm{C}$ for $30 \mathrm{~min}$ before clarification. Clarified lysates were diluted to a final salt concentration of $150 \mathrm{mM} \mathrm{KCl}$ with No-salt equilibration buffer $(20 \mathrm{mM}$ HEPES [pH 7.9], $0.5 \mathrm{mM}$ DTT, $0.05 \%$ [v/v] phosphatase inhibitor cocktails 2 and 3 (Sigma-Aldrich), 1X protease inhibitor (Roche)) and $2 \mathrm{mg}$ of protein was incubated with anti-FLAG M2 magnetic beads for $2 \mathrm{~h}$. Bead-substrate complexes were washed five times in wash buffer (20 mM HEPES [ $\mathrm{pH} 7.9$ ], $100 \mathrm{mM} \mathrm{KCl}, 0.5 \mathrm{mM}$ DTT, $1 \mathrm{X}$ protease inhibitor (Roche)) at $4{ }^{\circ} \mathrm{C}$ before elution with $3 \mathrm{x}$ FLAG peptide $(150 \mathrm{mM} \mathrm{NaCl}, 0.05 \%$ Tween, $150 \mathrm{ng} / \mu \mathrm{l}$ FLAG peptide (Sigma-Aldrich)). Eluates were boiled with Laemmli buffer and fractionated by SDS-PAGE.

FRAP. $53 \mathrm{BP}^{-/-}$MCF-7 cells were stably transduced with an mClover2-tagged fragment of 53BP1 encompassing amino acids 1107-1711 of the wild type protein. Two days before FRAP, $2.5 \times 10^{5}$ cells were seeded on a $35 \mathrm{~mm}$ glass-bottom plate. Cells were irradiated ( $5 \mathrm{~Gy}$ ) with an X-ray source to induce IRIF formation $2 \mathrm{~h}$ before imaging. Individual foci were selected and 10 prebleach images were acquired prior to a single bleach pulse with an Argon laser at a laser transmission of $100 \%$. Immediately after bleaching, 120 images were acquired in $5 \mathrm{~s}$ intervals at $0.5 \%$ laser intensity. All acquisition files were processed in ImageJ with the Stackreg plugin to account for nuclear migration in the recovery period. Regions of interest encompassing the bleached area, the entire nuclei and a section of background were selected and mean intensity values were quantified for each in ImageJ. These values were then normalized and fitted using the easyFRAP software ${ }^{52}$. All acquisitions were performed on a Leica SP8 SMD X confocal microscope.

Initial rate values were calculated based on curve of best fit generated in MATLAB according to the following equation.

$$
F(x)=a^{*} \exp \left(b^{*} x\right)+c^{*} \exp \left(d^{*} x\right)
$$

Curves of best fit were generated with the following coefficient values (with $95 \%$ confidence bounds), mC2-f53BP1: $a=0.3191$ (0.3083, 0.33), $b=0.0006296$ $(0.0005654,0.0006938), c=-0.3105(-0.32,-0.301), d=-0.009148(-0.009856$, $-0.00844)$, goodness of fit, $\mathrm{SSE}=0.00501$, R-square $=0.9964$, adjusted R-square $=0.9963, \mathrm{RMSE}=0.006572 ; \mathrm{mC} 2-\mathrm{f} 53 \mathrm{BP} 1 \mathrm{ODm}: a=0.4695(0.463,0.4759), b=$ $0.000618(0.0005858,0.0006501), c=-0.5312(-0.5555,-0.5069), d=-0.02533$ $(-0.02711,-0.02355)$, goodness of fit, $\mathrm{SSE}=0.01578, \mathrm{R}$-square $=0.9909$, adjusted $\mathrm{R}$-square $=0.9906, \mathrm{RMSE}=0.01167 ; \mathrm{mC} 2-\mathrm{f53BP} 1^{\mathrm{LC} 8 \mathrm{~m}}: a=0.4598(0.4451$, $0.4745), b=0.0004644(0.0004064,0.0005225), c=-0.4654(-0.4778,-0.4531)$, $d=-0.008194(-0.008687,-0.007701), \mathrm{SSE}=0.00528, \mathrm{R}$-square $=0.9979$, adjusted R-square $=0.9979$, RMSE $=0.006747$. Rates for $\mathrm{x}=10 \mathrm{~s}$ are presented in Fig. $3 e$. 
Expression and purification of 53BP1. Smt3-His 6 -tagged fragments (amino acids 1131-1292) of wild type (LC8-OD), OD-mutated (ODm-LC8), LC8-mutated (ODLC8m), double mutant (LC8m-ODm) 53BP1, and DYNLL1 were expressed from pET-17b in E. coli BL21(DE3). Cells were lysed by sonication in $20 \mathrm{mM}$ HEPES $\mathrm{pH}$ $7.5,250 \mathrm{mM} \mathrm{NaCl}$, and $0.25 \mathrm{mM}$ TCEP. Lysates were clarified by centrifugation and the supernatant was applied to a TALON IMAC column (Supplementary Fig. 2a) or Ni-NTA agarose beads (Fig. 3f). Bound complexes were washed with $20 \mathrm{mM}$ HEPES pH 7.5, $250 \mathrm{mM} \mathrm{NaCl}, 0.25 \mathrm{mM}$ TCEP, supplemented with $10 \mathrm{mM}$ imidazole and then eluted by increasing the imidazole concentration to $250 \mathrm{mM}$. Equal concentrations of eluate were then fractionated over a Superdex S200 column (Supplementary Fig. 2a).

In vivo tumourigenesis studies. All animal experiments were approved by the Animal Ethics Committee of The Netherlands Cancer Institute (Amsterdam, the Netherlands) and performed in accordance with the Dutch Act on Animal Experimentation (November 2014). KB1P4 tumour organoids were established previously and cultured in AdDMEM/F12 supplemented with 1 M HEPES (Sigma), GlutaMAX (Invitrogen), penicillin/streptomycin (Gibco), B27 (Gibco), $125 \mu \mathrm{M}$ $\mathrm{N}$-acetyl-L-cysteine (Sigma), $50 \mathrm{ng} / \mathrm{mL}$ murine epidermal growth factor (Invitrogen) (Duarte et al. ${ }^{43}$ ). Tumour organoid transduction was performed by spinoculation with pLentiCRISPRv2 lentiviral constructs in which either no gRNA or a gRNA targeting ASCIZ was cloned. Following puromycin selection, modified organoids were collected, incubated with TripLE at $37^{\circ} \mathrm{C}$ for $5^{\prime}$, dissociated into single cells, washed in PBS, resuspended in tumour organoid medium and mixed in a 1:1 ratio of tumour organoid suspension and BME in a cell concentration of $10^{4}$ cells per $40 \mu \mathrm{l}$. Subsequently, $10^{4}$ cells were transplanted in the fourth right mammary fat pad of 6-9-week-old female NMRI nude mice. Mammary tumour size was measured by caliper measurements and tumour volume was calculated $\left(0.5 \times\right.$ length $\times$ width $\left.^{2}\right)$. Treatment of tumour bearing mice was initiated when tumours reached a size of $50-100 \mathrm{~mm}^{3}$, at which point mice were stratified into the untreated $(n=8)$ or olaparib treatment group $(n=8)$. Olaparib was administered at $100 \mathrm{mg} / \mathrm{kg}$ intraperitoneally for 80 consecutive days. Animals were sacrificed with $\mathrm{CO}_{2}$ when the tumour reached a volume of $1500 \mathrm{~mm}^{3}$. Sample sizes were not pre-determined and no blinding or randomization was employed during analysis.

Quantification and statistical analysis. Prism 6 (GraphPad Software Inc.) was used for statistical analysis and production of all graphs and dot plots. The relevant statistical methods and measures of significance for each experiment are detailed in the figure legends. Cell Profiler (Broad Institute) was used for automated quantitation of immunofluorescence data. Normalization of FRAP recovery curves was performed using the easyFRAP software and curves of best fit were generated in MATLAB.

\section{Data availability}

The source data underlying Figs. $1 \mathrm{~d}-\mathrm{f}, 2 \mathrm{~b}$ and d, $3 \mathrm{~b}-\mathrm{d}$ and f, $4 \mathrm{~d}, \mathrm{f}$ and $\mathrm{g}, 5 \mathrm{a}$ and $\mathrm{b}$, and Supplementary Figures $1 \mathrm{c}$ and d, $2 \mathrm{~b}-\mathrm{e}, 3 \mathrm{a}-\mathrm{d}, 4 \mathrm{~b}-\mathrm{f}, 5 \mathrm{c}$ and $\mathrm{e}$, and $6 \mathrm{a}$ and $\mathrm{c}$ are provided as a Source Data file. All other original data and code that supports the findings of this study are available from the corresponding author upon reasonable request.

Received: 14 June 2018 Accepted: 30 November 2018

Published online: 17 December 2018

\section{References}

1. Jackson, S. P. \& Bartek, J. The DNA-damage response in human biology and disease. Nature 461, 1071-1078 (2009).

2. Dudley, D. D., Chaudhuri, J., Bassing, C. H. \& Alt, F. W. Mechanism and control of $\mathrm{V}(\mathrm{D}) \mathrm{J}$ recombination versus class switch recombination: similarities and differences. Adv. Immunol. 86, 43-112 (2005).

3. Ciccia, A. \& Elledge, S. J. The DNA damage response: making it safe to play with knives. Mol. Cell 40, 179-204 (2010).

4. Hustedt, N. \& Durocher, D. The control of DNA repair by the cell cycle. Nat. Cell Biol. 19, 1 (2016)

5. Chapman, J. R., Taylor, M. R. \& Boulton, S. J. Playing the end game: DNA double-strand break repair pathway choice. Mol. Cell 47, 497-510 (2012).

6. Panier, S. \& Boulton, S. J. Double-strand break repair: 53BP1 comes into focus. Nat. Rev. Mol. Cell Biol. 15, 7-18 (2014).

7. Botuyan, M. V. et al. Structural basis for the methylation state-specific recognition of histone H4-K20 by 53BP1 and Crb2 in DNA repair. Cell 127, 1361-1373 (2006).

8. Fradet-Turcotte, A. et al. 53BP1 is a reader of the DNA-damage-induced H2A Lys 15 ubiquitin mark. Nature 499, 50-54 (2013).
9. Chapman, J. R. et al. RIF1 is essential for 53BP1-dependent nonhomologous end joining and suppression of DNA double-strand break resection. Mol. Cell 49, 858-871 (2013).

10. Escribano-Díaz, C. et al. A cell cycle-dependent regulatory circuit composed of 53BP1-RIF1 and BRCA1-CtIP controls DNA repair pathway choice. Mol. Cell 49, 872-883 (2013).

11. Zimmermann, M., Lottersberger, F., Buonomo, S. B., Sfeir, A. \& de Lange, T. 53BP1 regulates DSB repair using Rif1 to control 5' end resection. Science 339, 700-704 (2013).

12. Ward, I. M. et al. 53BP1 is required for class switch recombination. J. Cell Biol. 165, 459-464 (2004).

13. Manis, J. P. et al. 53BP1 links DNA damage-response pathways to immunoglobulin heavy chain class-switch recombination. Nat. Immunol. $\mathbf{5}$, 481 (2004).

14. Di Virgilio, M. et al. Rif1 prevents resection of DNA breaks and promotes immunoglobulin class switching. Science 339, 711-715 (2013).

15. Hakim, O. et al. DNA damage defines sites of recurrent chromosomal translocations in B lymphocytes. Nature 484, 69 (2012).

16. Dimitrova, N., Chen, Y.-C. M., Spector, D. L. \& de Lange, T. 53BP1 promotes non-homologous end joining of telomeres by increasing chromatin mobility. Nature 456, 524 (2008).

17. Sfeir, A. \& De Lange, T. Removal of shelterin reveals the telomere endprotection problem. Science 336, 593-597 (2012).

18. Cao, L. et al. A selective requirement for 53BP1 in the biological response to genomic instability induced by Brcal deficiency. Mol. Cell 35, 534-541 (2009).

19. Bunting, S. F. et al. 53BP1 inhibits homologous recombination in Brcaldeficient cells by blocking resection of DNA breaks. Cell 141, 243-254 (2010).

20. Jaspers, J. E. et al. Loss of 53BP1 causes PARP inhibitor resistance in Brcalmutated mouse mammary tumors. Cancer Discov. 3, 68-81 (2013).

21. Xu, G. et al. REV7 counteracts DNA double-strand break resection and affects PARP inhibition. Nature 521, 541-544 (2015).

22. Cuella-Martin, R. et al. 53BP1 integrates DNA repair and p53-dependent cell fate decisions via distinct mechanisms. Mol. Cell 64, 51-64 (2016).

23. Fong, C. S. et al. 53BP1 and USP28 mediate p53-dependent cell cycle arrest in response to centrosome loss and prolonged mitosis. eLife 5, e16270 (2016).

24. Lambrus, B. G. et al. A USP28-53BP1-p53-p21 signaling axis arrests growth after centrosome loss or prolonged mitosis. J. Cell Biol. 214, 143-153 (2016).

25. Meitinger, F. et al. 53BP1 and USP28 mediate p53 activation and G1 arrest after centrosome loss or extended mitotic duration. J. Cell Biol. 214, 155-166 (2016).

26. Bothmer, A. et al. Regulation of DNA end joining, resection, and immunoglobulin class switch recombination by 53BP1. Mol. Cell 42, 319-329 (2011).

27. Lottersberger, F., Bothmer, A., Robbiani, D. F., Nussenzweig, M. C. \& de Lange, $\mathrm{T}$. Role of 53BP1 oligomerization in regulating double-strand break repair. Proc. Natl. Acad. Sci. 110, 2146-2151 (2013).

28. Zgheib, O., Pataky, K., Brugger, J. \& Halazonetis, T. D. An oligomerized 53BP1 tudor domain suffices for recognition of DNA double-strand breaks. Mol. Cell. Biol. 29, 1050-1058 (2009).

29. Wilson, M. D. et al. The structural basis of modified nucleosome recognition by 53BP1. Nature 536, 100-103 (2016).

30. Iwabuchi, K. et al. Potential role for 53BP1 in DNA end-joining repair through direct interaction with DNA. J. Biol. Chem. 278, 36487-36495 (2003)

31. Huyen, Y. et al. Methylated lysine 79 of histone H3 targets 53BP1 to DNA double-strand breaks. Nature 432, 406 (2004).

32. Lo, K. W.-H. et al. The 8-kDa dynein light chain binds to $\mathrm{p} 53$-binding protein 1 and mediates DNA damage-induced p53 nuclear accumulation. J. Biol. Chem. 280, 8172-8179 (2005)

33. King, S. M. Dynein-independent functions of DYNLL1/LC8: redox state sensing and transcriptional control. Sci. Signal. 1, pe51-pe51 (2008).

34. Barbar, E. Dynein light chain LC8 is a dimerization hub essential in diverse protein networks. Biochemistry 47, 503-508 (2008).

35. Matsuoka, S. et al. ATM and ATR substrate analysis reveals extensive protein networks responsive to DNA damage. Science 316, 1160-1166 (2007).

36. Clark, S. et al. Multivalency regulates activity in an intrinsically disordered transcription factor. eLife 7, e36258 (2018).

37. King, A. et al. Dynein light chain regulates adaptive and innate B cell development by distinctive genetic mechanisms. PLoS. Genet. 13, e1007010 (2017).

38. Jurado, S. et al. ATM substrate Chk2-interacting Zn2 + finger (ASCIZ) is a bifunctional transcriptional activator and feedback sensor in the regulation of dynein light chain (DYNLL1) expression. J. Biol. Chem. 287, 3156-3164 (2012).

39. Jurado, S. et al. The Zinc-finger protein ASCIZ regulates B cell development via DYNLL1 and Bim. J. Exp. Med. 209, 1629-1639 (2012).

40. Loizou, J. I. et al. ATMIN is required for maintenance of genomic stability and suppression of B cell lymphoma. Cancer Cell. 19, 587-600 (2011). 
41. Brinkman, E. K., Chen, T., Amendola, M. \& van Steensel, B. Easy quantitative assessment of genome editing by sequence trace decomposition. Nucleic Acids Res. 42, e168-e168 (2014).

42. Wong, D. M. et al. The transcription factor ASCIZ and its target DYNLL1 are essential for the development and expansion of MYC-driven B cell lymphoma. Cell Rep. 14, 1488-1499 (2016).

43. Duarte, A. A. et al. BRCA-deficient mouse mammary tumor organoids to study cancer-drug resistance. Nat. Methods 15, 134 (2018).

44. Brummelkamp, T. R. et al. An shRNA barcode screen provides insight into cancer cell vulnerability to MDM2 inhibitors. Nat. Chem. Biol. 2, 202-206 (2006).

45. Difilippantonio, S. et al. 53BP1 facilitates long-range DNA end-joining during V (D) J recombination. Nature 456, 529-533 (2008).

46. Ghezraoui, H. et al. 53BP1 cooperation with the REV7-shieldin complex underpins DNA structure-specific NHEJ. Nature 560, 122-127 (2018).

47. Ward, I. M., Minn, K., van Deursen, J. \& Chen, J. p53 Binding Protein 53BP1 Is Required for DNA Damage Responses and Tumor Suppression in Mice. Mol. Cell. Biol. 23, 2556 (2003).

48. Noordermeer, S. M. et al. The shieldin complex mediates 53BP1-dependent DNA repair. Nature 15, 1-25 (2018).

49. Dev, H. et al. Shieldin complex promotes DNA end-joining and counters homologous recombination in BRCA1-null cells. Nat. Cell Biol. 66, 129-21 (2018).

50. Pfaffl, M. W. A new mathematical model for relative quantification in realtime RT-PCR. Nucleic Acids Res. 29, e45-e45 (2001).

51. Pone, E. J. et al. BCR-signalling synergizes with TLR-signalling for induction of AID and immunoglobulin class-switching through the non-canonical NFкB pathway. Nat. Commun. 3, 767 (2012).

52. Rapsomaniki, M. A. et al. easyFRAP: an interactive, easy-to-use tool for qualitative and quantitative analysis of FRAP data. Bioinformatics $\mathbf{2 8}$ 1800-1801 (2012).

\section{Acknowledgements}

This study was funded by Cancer Research UK Career Development Fellowship (CRUK CDF; C52690/A19270) awarded to J.R.C., that provides salary support for C.O. and J.R. B.; a Nuffield Department of Medicine Prize DPhil studentship to R.C.M.; CRUK CDF funding to ANB (C29215/A20772); CRUK grant (C302/A24386) to AWO; grants from Worldwide Cancer Research (WCR 16-0156) and the National Health and Medical Research Council of Australia (NHMRC 1139099) to JH; financial support also came from the Dutch Cancer Society (KWF 2014-6532 to S.R. and J.J.), the Netherlands Organization for Scientific Research (VICI 91814643, NGI 93512009, Cancer Genomics Netherlands and a National Roadmap grant for Large-Scale Research Facilities to J.J.), the Swiss National Science Foundation (310030_156869 to S.R.), the Swiss Cancer League (KLS-4282-08-2017 to S.R.), and the European Union (ERC StG 311565 to J.J.L.J., ERC CoG-681572 to S.R.). The Wellcome Centre for Human Genetics is supported by Wellcome core award 090532/Z/09/Z. St. Vincent's Institute of Medical Research is supported by NHMRC Independent Research Institutes Infrastructure Support and Victorian State Government Operational Infrastructure Support grants. The authors thank A. Nussenzweig and E. Callen-Moreau for advice/reagents, S. Snellenberg for cellcycle lysates, and all laboratory members for discussions and critique. We also thank C. Green (Chromosome Dynamics core) for expertise and assistance with FRAP experiments. We thank D. Durocher and D. Chowdhury for discussing unpublished results, and credit D. Durocher and S. Noordermeer for sharing DYNLL1-targeting CRISPR reagents and for initiating the mouse tumour studies with S.R. and J.J.

\section{Author contributions}

J.R.B. co-wrote the manuscript, prepared the figures and designed, performed and analysed the majority of experiments, with assistance from R.C.M., C.O., A.W.O., K.B. and A.N.B. In vivo tumour models were performed by M.B. under the supervision of J.J. and SR. CSR experiments involving Dynll1 and Asciz-mutant mice were performed by R. L. under the supervision of J.H. A.B.H. provided computational analysis. J.R.C. conceived and supervised the study and co-wrote the manuscript.

\section{Additional information}

Supplementary Information accompanies this paper at https://doi.org/10.1038/s41467 018-07855-x.

Competing interests: The authors declare no competing interests.

Reprints and permission information is available online at http://npg.nature.com/ reprintsandpermissions/

Journal peer review information: Nature Communications thanks the anonymous reviewers for their contributions to the peer review of this work. Peer Reviewer Reports are available.

Publisher's note: Springer Nature remains neutral with regard to jurisdictional claims in published maps and institutional affiliations.

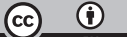

Open Access This article is licensed under a Creative Commons Attribution 4.0 International License, which permits use, sharing, adaptation, distribution and reproduction in any medium or format, as long as you give appropriate credit to the original author(s) and the source, provide a link to the Creative Commons license, and indicate if changes were made. The images or other third party material in this article are included in the article's Creative Commons license, unless indicated otherwise in a credit line to the material. If material is not included in the article's Creative Commons license and your intended use is not permitted by statutory regulation or exceeds the permitted use, you will need to obtain permission directly from the copyright holder. To view a copy of this license, visit http://creativecommons.org/ licenses/by/4.0/.

(C) The Author(s) 2018 